\title{
Redescription of seven species of eriophyoid mites from bamboo (Poaceae, Bambuseae) in Thailand
}

\author{
Ploychompoo KONVIPASRUANG $^{1}$, Angsumarn ChANDRAPATYA $^{1} *$ and J.W. AMRINE, Jr. ${ }^{2}$ \\ ${ }^{1}$ Department of Entomology, Kasetsart University, Chatuchak, Bangkok 10900, Thailand. \\ ${ }^{2}$ Division of Plant and Soil Sciences, P.O.Box 6108, College of Agriculture and Forestry, West \\ Virginia University, Morgantown, WV 26506-6108, USA
}

(Received 15 January 2012; Accepted 31 July 2012)

\begin{abstract}
Seven species of eriophyoid mites previously described on bamboo plants in Thailand by Chandrapatya and Boczek are redescribed and/or reassigned. Phaicus is described as a new genus with Tegolopus [sic] bambusae ChannaBasavanna, 1966 designated as the type species and Abacarus panticis Keifer, 1977 and Aculodes ventricosae Chandrapatya, 1998 as synonyms. Aculus asperi (Chandrapatya and Boczek, 2002) is reassigned to Abacarus and redescribed. Abacarus klaengensis is a new name to replace Abacarus bambusae (Boczek, 1998) which is a junior homonym of Abacarus bambusae Kuang and Zhuo, 1987. Knorella blumeanae Xue and Zhang, 2009 is emended from Knorella blumcanae Xue and Zhang, 2009 due to misspelling of host plant. Neocatarhinus namtai is a new name to replace Neocatarhinus bambusae (Boczek, 1998), new combination,which is a junior homonym of Neocatarhinus bambusae Kuang and Hong, 1990. Kolacarus bambusae Boczek, 1998 and Knorella thailandica Hong, 1996, are redescribed. Males were described when found. Host lists and collecting data are corrected and new host plants with distribution have been added.
\end{abstract}

Key words: bamboo, Eriophyoidea, Eriophyidae, Diptilomiopidae, Abacarus, Phaicus, Neocatarhinus, Kolacarus, Knorella, Thailand.

\section{INTRODUCTION}

Bamboo plants are mostly found in the tropics, and are especially common and diverse in Thailand, where many genera have been reported such as Bambusa, Dendrocalamus, Thyrsostachys, Schizostachyum, Melocalamus, Melocanna and Gigantochloa. Bamboos are infested with many species of plant feeding mites including eriophyoid mites. Keifer (1975) was the first to identify and describe eriophyoid mites on bamboo in Thailand. During 1975-1978, he described four new species of eriophyoid mites associated with Thai bamboo, namely, Abacarus panticis Keifer, 1977 (junior synonym of Abacarus bambusae (ChannaBasavanna, 1966) on

* Corresponding author: E-mail; agramc@ku.ac.th DOI: 10.2300 /acari.21.67 
Bambusa ventricosa McClure, Bangkhen, Bangkok; Diptiloplatus megagrastis Keifer, 1975, Knorella gigantochloae Keifer, 1975 and Tegolophus bangkokensis Keifer, 1978 on Gigantochloa ligulata Gamble, Bangkok, Thailand.

Since then, extensive collections of eriophyoid mites in Thailand were made during 19852002 by Chandrapatya, and a series of studies of eriophyoid mites in Thailand were published by Chandrapatya and Boczek, including seven new species of eriophyoid mites feeding on bamboo (Boczek \& Chandrapatya, 1978; Chandrapatya \& Boczek, 1993, 2002).

The aim of this paper is to present new information on the reported species by Chandrapatya and Boczek using the revised keys to world genera of Eriophyoidea (Amrine et al., 2003) for identification. The repeated use of the species designation, bambusae for names of eriophyoid mites, (there are 24 in the literature at present) has resulted in numerous junior homonyms when correct generic assignments are made. Therefore, authors should avoid further use of the name "bambusae". In addition to the mites redescribed here, several new species will be described in future papers.

All holotypes, but one species, were lost or misplaced in the collection of the Warsaw Agricultural University, Poland. Illustrations, measurements and new descriptions are mainly based on paratypes and other specimens not included in the type series except for Kolacarus bambusae where the holotype is still available. All measurements are given in micrometers $(\mu \mathrm{m})$. For females, each measurement of the holotype or selected paratype (neotype) precedes the corresponding range for the paratypes, unless the same sizes are recorded for both holotype and paratype. The count of dorsal annuli starts from the rear shield margin; the count of ventral annuli starts from the first lateral annulus at the lateral prodorsal shield margin. All measurements were performed according to de Lillo et al. (2010). All type specimens are deposited in the collection of Department of Entomology, Kasetsart University, Bangkok, Thailand.:

\section{Setal notation used in this paper}

Prodorsal Shield:

sc scapular seta (dorsal setae)

Opisthosoma:

$c 2 \quad$ opisthosomal seta $c 2$ (lateral setae)

$d \quad$ opisthosomal seta $d$ (1st ventral setae)

$e \quad$ opisthosomal seta $e$ (2nd ventral setae)

$f \quad$ opisthosomal seta $f$ (3rd ventral setae)

$h 1 \quad$ opisthosomal seta $h 1$ (accessory setae)

$h 2 \quad$ opisthosomal seta $h 2$ (caudal setae)

Pedipalp:

ep pedipalp coxal seta (basal setae)

$d \quad$ dorsal pedipalp genual seta (antapical setae)

$v \quad$ subapical pedipalp tarsal seta (sensory peg, papilla)

Coxisternal plates:

1a proximal seta on coxisternum I (2nd coxal setae)

$1 b$ anterior seta on coxisternum I (1st coxal setae) 


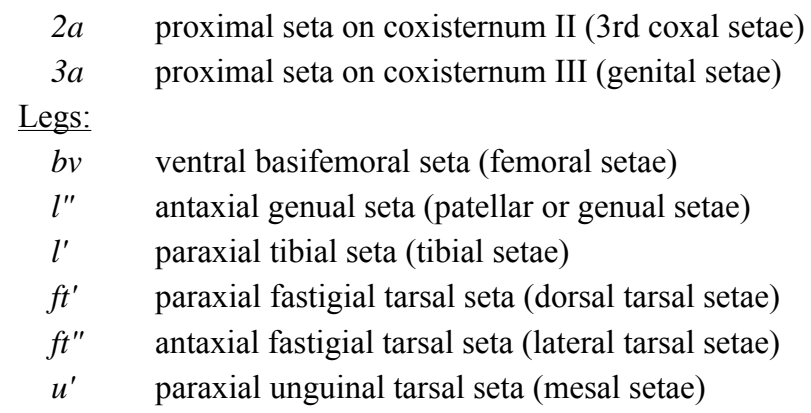

\title{
DESCRIPTIONS
}

\author{
Family Eriophyidae Nalepa, 1898 \\ Subfamily Phyllocoptinae Nalepa, 1892 \\ Tribe Anthocoptini Amrine \& Stasny, 1994 \\ Phaicus Konvipasruang, Chandrapatya and Amrine gen. nov.
}

(Fig. 1)

Type species: Tegolopus bambusae ChannaBasavanna, 1966

ChannaBasavanna, 1966: 122-123, pl. 31-32.

Adult female. (based on mites from Thailand originally described as Aculodes ventricosae Chandrapatya, 1998): body slightly vermiform. Gnathosoma projecting slightly downwards, dorsal pedipalp genual seta $d$ present. Prodorsal shield subtriangular, slightly elongate posteriorly, with small, pointed anterior triangular prodorsal shield frontal lobe over gnathosoma; scapular tubercles situated on rear shield margin, directing scapular setae $s c$ divergently to the rear. Legs with all setae present; empodium simple. Coxisterna I and II with all setae present. Opisthosomal annuli microtuberculate. Dorsal annuli forming three weak longitudinal ridges, middorsal ridge equal to subdorsal ridges. Last 10-11 dorsal annuli with smaller microtubercles. All opisthosomal setae present. Genital coverflap with several longitudinal ridges.

Diagnosis. This new genus is close to Tegolophus. Phaicus has a weak prodorsal shield frontal lobe; opisthosoma with three weak longitudinal ridges, middorsal ridge equal to subdorsal ridges. Tegolophus has a strong, rounded prodorsal shield frontal lobe; middorsal ridge stronger than subdorsal ridges and fading caudally.

Etymology. The generic name is after the Thai common name of the host plant, bamboo (Phai+ -cus, shortened form of Latin acarus, a mite).

Discussion and Diagnosis. This mite represents a new genus in the Phyllocoptinae, Anthocoptini; it belongs to a group of genera that have three dorsal opisthosomal ridges [which rules out Aculodes, Aculops and Aculus, etc.]; see Revised keys to world genera of Eriophyoidea (Amrine et al., 2003). Mites assigned to Abacarus are elongate and vermiform, usually with a prominent acuminate prodorsal shield frontal lobe. The opisthosomal dorsal ridge fades before dorsal annuli above seta $f$ into a dorsal furrow or trough. The dorsal annuli of most species of Abacarus bear small, dense microtubercles along the posterior margin of each annulus. Tegolophus is fusiform, with a shorter body length, with a stout, rounded prodorsal shield frontal 
lobe, and with a prominent middorsal ridge that extends to or beyond dorsal annuli above seta $f$. If the middorsal ridge ends in a shallow trough [very rare] then the dorsal opisthosomal annuli are smooth or bear larger, less dense microtubercles compared to Abacarus. Phaicus has an elongate, somewhat vermiform body; the prodorsal shield frontal lobe is weaker, pointed anteriorly; the middorsal opisthosomal ridge is weak and extends to, or beyond dorsal annuli above seta $f$; the dorsal annuli bear normal microtubercles in the three "species" described from bamboo, two of which, panticis and ventricosae are now synonyms.

We initially found three species that we included in Phaicus, but after careful analyses, we discovered that these were three descriptions of the same species. The first, and the genotype, was originally described by ChannaBasavanna in 1966 as Tegolopus [sic] bambusae ChannaBasavanna, 1966, now Phaicus bambusae (ChannaBasavanna, 1966). The other two species are Abacarus panticis Keifer, 1977, and Aculodes ventricosae Chandrapatya, 1998; none of the three mites fit into the genera into which they were originally placed.

Because of the confusion surrounding the name Abacarus bambusae, we studied Abacarus bambusae Kuang \& Zhuo, 1987, since Huang (2001) indicated that it was a junior synonym to “Abacarus bambusae ChannaBasavanna, 1966" (parentheses left out in Huang's paper). The mite described by Kuang \& Zhuo has 6 rays to the empodium, a rounded prodorsal shield frontal lobe, middorsal ridge ending in a trough before dorsal annuli above seta $f$, a very different sculpturing of the prodorsal shield, and coxae sculpted with numerous sinuate lines. It is a distinct, separate species in Abacarus. Huang (2001) illustrated the mite that he labeled as "Abacarus bambusae ChannaBasavanna, 1966", instead of Tegolopus [sic] bambusae which it very closely resembles. This drawing depicts a very similar, but slightly different prodorsal shield from that of Phaicus bambusae (ChannaBasavanna). In addition, his drawing of the mite is more tapered, and it has a rounded prodorsal shield frontal lobe. His mite species needs more careful study.

\section{Redescription of Tegolopus [sic] bambusae ChannaBasavanna, 1966, based on specimens collected in Thailand as Abacarus ventricosae (Chandrapatya, 1998).}

FEMALE (Fig.1) (n=13). Body slightly vermiform, 180 (140-180) long, 55 (43-55) wide, (46-56) thick; color in life red. Gnathosoma: projecting slightly downwards, 20 (20-24) long; pedipalp coxal seta ep 4 (4-5) long, dorsal pedipalp genual seta $d 5$ (4-6) long. Chelicerae 17 (17-22) long, almost straight. Prodorsal shield: 35 (34-38) long, 42 (40-45) wide, subtriangular, slightly elongate posteriorly, ornamented with a few granules. Scapular setae sc 20 (16-30) long, on scapular tubercles 22 (22-25) apart, situated on rear shield margin, directed divergently to the rear. Shield design consists of median, admedian, submedian lines and three oblique lateral lines on each side. Prodorsal shield frontal lobe triangular, pointed anteriorly, extending over gnathosoma, 3 (3-4) long. Legs: with all setae present. Leg I 27 (20-31) long; femur 10 (7-10) long, ventral basifemoral seta $b v 10$ (8-15) long; genu 5 (4-6) long, antaxial genual seta $l$ " 30 (20-34) long; tibia 6 (5-7) long, paraxial tibial seta $l^{\prime} 10$ (6-15) long; tarsus 6 (6-7) long, antaxial fastigial tarsal seta $f t^{\prime \prime} 23$ (18-25) long, paraxial fastigial tarsal seta $f t^{\prime} 22$ (19-23) long, paraxial unguinal tarsal seta $u^{\prime} 4$ (3-5) long, solenidion slightly curved, evenly tapered and truncate apically, 7 (6-8) long; empodium simple, 6 (5-8) long, 6-rayed. Leg II 25 (21-28) long; femur 10 (7-11) long, ventral basifemoral seta $b v 10$ (10-25) long; genu 4 (4-5) long, antaxial genual 


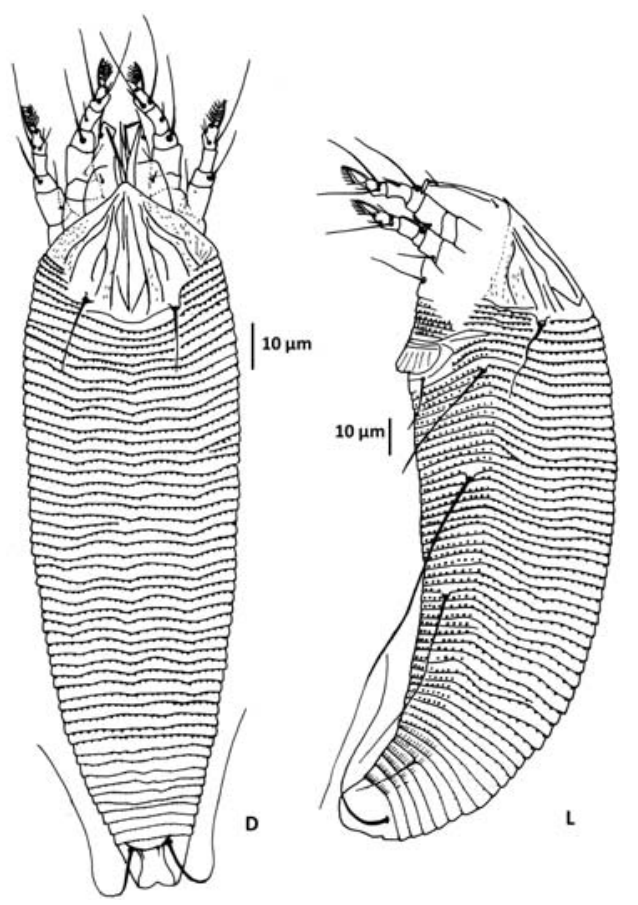

Fig. 1. Phaicus ventricosae (Chandrapatya)

seta $l^{\prime \prime} 12$ (8-13) long; tibia 5 (5-6) long; tarsus 6 (5-7) long, antaxial fastigial tarsal seta $\mathrm{ft}^{\prime \prime} 22$ (14-22) long, paraxial fastigial tarsal seta $f t^{\prime} 7$ (6-10) long, paraxial unguinal tarsal seta $u^{\prime} 3$ (35) long, solenidion as in leg I, 6 (6-8) long; empodium simple, 6 (5-8) long, 6-rayed. Coxisternal plates: both coxisterna with numerous short lines. Internal coxisternal apodeme a line 9 (710) long. Anterior seta on coxisternum I $1 b 6$ (5-11) long, 12 (10-12) apart; proximal seta on coxisternum I $1 a 18$ (14-25) long, 10 (8-10) apart; proximal seta on coxisternum II $2 a 28$ (2236) long, 22 (20-22) apart; distance between setae $1 b$ and $1 a 8$ (8-9) long; coxigenital semiannuli 6 (6-7), microtuberculate. Opisthosoma: with 53 (46-59) dorsal annuli, each annulus with small, triangular microtubercles, last 10 (10-11) annuli with smaller microtubercles. Ventral opisthosoma with 55 (50-62) annuli, with rounded microtubercles; dorsal microtubercles situated behind rear margin of annuli, ventral microtubercles situated ahead of rear margin of each annulus. Microtubercles elongated on the last 7-9 ventral annuli. First 32-46 dorsal annuli forming three weak longitudinal ridges of similar length. Opisthosomal seta $c 232$ (20-35) long, 48 (40-48) apart, on annulus 8 (7-11); opisthosomal seta $d 98$ (60-98) long, 35 (29-35) apart, on annulus 19 (18-24); opisthosomal seta e 52 (40-52) long, 20 (15-20) apart, on annulus 32 (2937 ); opisthosomal seta $f 20$ (18-27) long, 19 (17-22) apart, on annulus 51 (45-57), or 5 (5-7) from the rear. Opisthosomal seta $h 253$ (22-53) long; opisthosomal seta $h 12$ (2-3) long. Genital coverflap: 22 (18-22) wide, 17 (14-17) long, with 12-14 longitudinal ridges; proximal seta on coxisternum III $3 a 20$ (19-21) long, 20 (18-20) apart. 

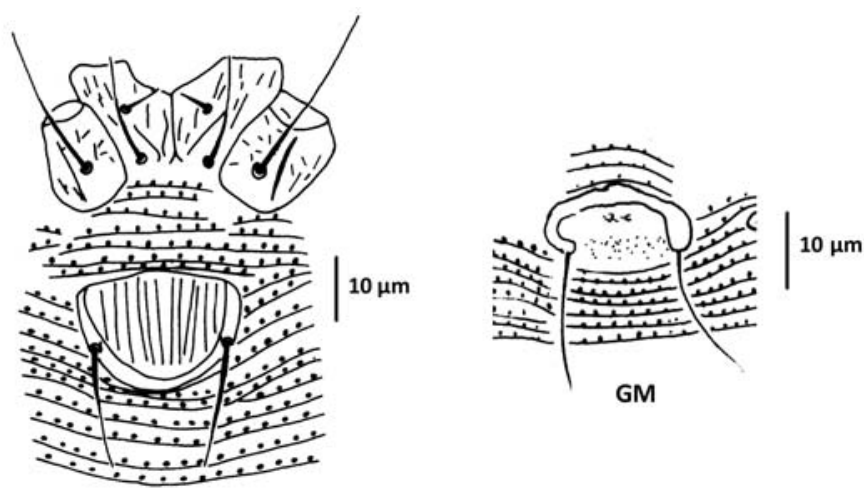

GF
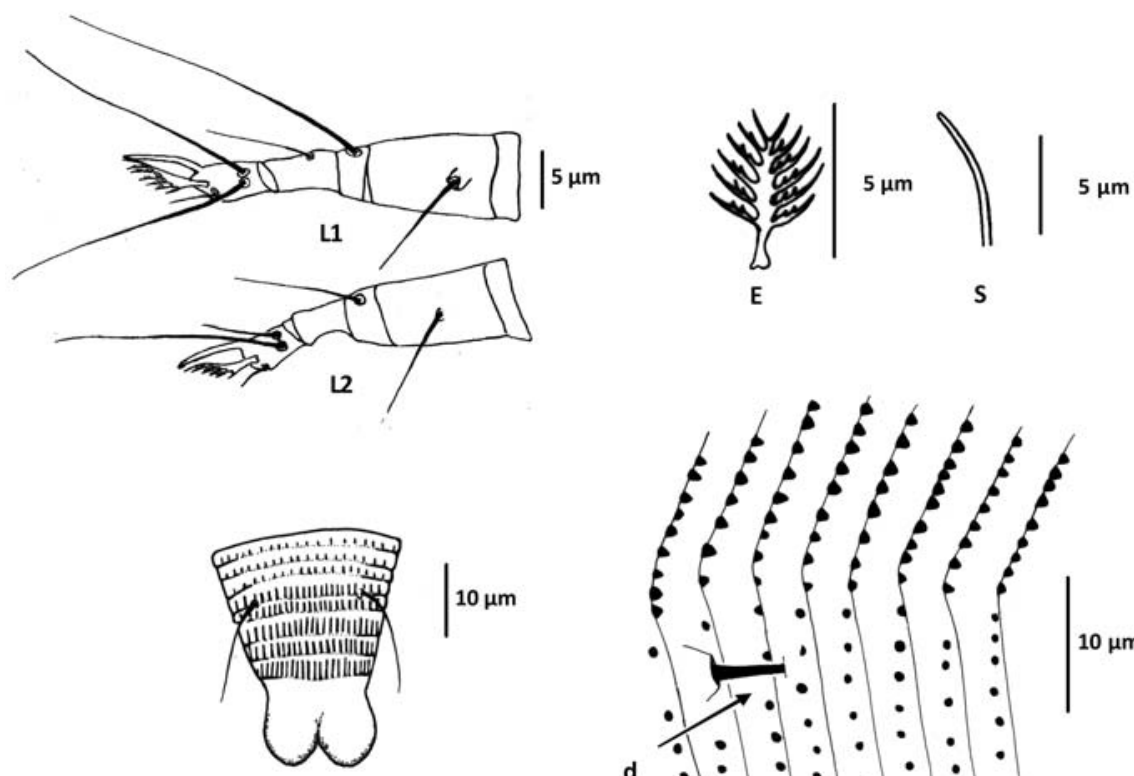

CS

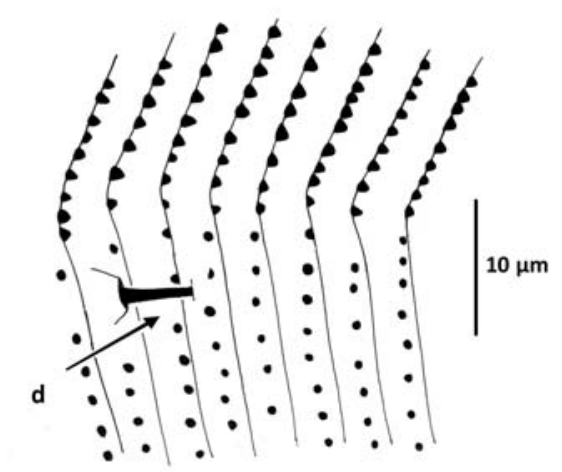

LO

Fig. 1. (Continue) Phaicus ventricosae (Chandrapatya)

MALE $(\mathrm{n}=2$, slide \# 1904). Smaller than female, 121-138 long, 45 wide. Gnathosoma: 1819 long; pedipalp coxal seta ep 3 long, dorsal pedipalp genual seta $d 5$ long. Chelicerae 14-19 long. Prodorsal shield: 32-33 long, 40-41 wide, similar to female. Scapular setae sc 15-18 long, on scapular tubercles 22-24 apart. Prodorsal shield frontal lobe 2-3 long. Legs: leg I 26-30 long; femur 8-9 long, ventral basifemoral seta $b v$ 8-10 long; genu 4-5 long, antaxial genual seta $l$ " 2425 long; tibia 5-6 long, paraxial tibial seta $l^{\prime}$ 6-7 long; tarsus 5 long, antaxial fastigial tarsal seta $f t^{\prime \prime} 18$ long, paraxial fastigial tarsal seta $f t^{\prime}$ 15-18 long, paraxial unguinal tarsal seta $u^{\prime} 3$ long, 
solenidion slightly curved, tapered apically, 6 long; empodium simple, 6 long, 6-rayed. Leg II 23-24 long; femur 8-9 long, ventral basifemoral seta $b v$ 18-20 long; genu 4-5 long, antaxial genual seta $l$ " 10 long; tibia 4-5 long; tarsus 5-6 long, antaxial fastigial tarsal seta $f t^{\prime \prime} 18-20$ long, paraxial fastigial tarsal seta $f t^{\prime} 7$ long, paraxial unguinal tarsal seta $u^{\prime}$ 3-4 long, solenidion slightly curved, unknobbed, 7 long; empodium simple, 6 long, 6-rayed. Coxisternal plates: both coxisterna with numerous short lines. Internal coxisternal apodeme a line 5-7 long. Anterior seta on coxisternum I $1 b 10$ long, 10 apart; proximal seta on coxisternum I la 18-22 long, 7-8 apart; proximal seta on coxisternum II $2 a$ 30-31 long, 17-18 apart; distance between setae $1 b$ and $1 a$ 6-7 long; coxigenital semiannuli 6-7, microtuberculate. Opisthosoma: with 48-49 dorsal annuli, 53-57 ventral annuli. Opisthosomal seta $c 230$ long, 36-38 apart, on annulus 9; opisthosomal seta $d$ 50-55 long, 28 apart, on annulus 19-20; opisthosomal seta $e$ 40-45 long, 15 apart, on annulus 33; opisthosomal seta $f 20$ long, 18 apart, on annulus 45 (45-56), or 5 from the rear. Opisthosomal seta $h 250$ long; opisthosomal seta $h 12$ long. Genitalia: 12-14 wide, proximal seta on coxisternum III $3 a$ 13-14 long, 13-15 apart.

Etymology: the species name was based on the host plant generic name, Bambusa.

Type material: female and male paratypes on 8 slides labeled \#213.1 and 6 slides labeled $\# 1904$.

Host plant and locality of bambusae, ChannaBassavanna, 1966: Bambusa vulgaris Schrad. ex J. C. Wendl. (as Bambusa vulgaris Schrad.), Agricultural Research Station, Mysore, Karnataka, India, 23 June, 1962.

Host plant and locality of Aculodes ventricosae Chandrapatya, 1998: collected from leaves of Bambusa ventricosa McClure (Phai namtao, Buddha's belly bamboo), Poaceae, Bambuseae, Amphoe Kamphaeng Saen, Nakhon Pathom Province, Thailand, 2 November 1996, collected by A. Chandrapatya; and from Bambusa sp. (Phai), Poaceae, Bambuseae, Amphoe Mueang, Phra Nakhon Si Ayutthaya Province, Thailand, 24 February 2000, collected by A. Chandrapatya.

Other hosts and distribution: Bambusa sp. (Phai), Poaceae, Bambuseae, Khet Dusit, Bangkok, Thailand, 3 July 1998, collected by A. Chandrapatya (on two slides labeled \#846).

Relation to host: vagrant on the lower leaf surface. No apparent damage was observed.

Abacarus asperi (Chandrapatya \& Boczek, 2002): new combination.

Bamboocarus asperi Chandrapatya \& Boczek, 2002: 144-147, f. 4.

Aculus asperi (Chandrapatya \& Boczek, 2002); Amrine et al., 2003: 121.

Aculus asperi (Chandrapatya \& Boczek, 2002); Xue \& Zhang, 2009: 81.

\section{Redescription:}

FEMALE (Fig. 2) (n=9). Body fusiform, 170 (164-195) long, 59 (58-60) wide, 55 (5060) thick; color in life red. Gnathosoma: projecting downwards, 25 (22-26) long; pedipalp coxal seta ep 2-3 long, dorsal pedipalp genual seta $d 6$ (5-8) long. Chelicerae 19 (18-21) long, almost straight. Prodorsal shield: 54 (52-58) long, 52 (52-58) wide, pear-like. Scapular setae sc 4 (3-5) long, on scapular tubercles 35 (35-38) apart, situated on rear shield margin, directed divergently to the rear. Shield surface smooth. Prodorsal shield frontal lobe broad, rounded anteriorly, extending over gnathosoma, 12 (9-12) long. Legs: with all setae present. Leg I 29 (28-31) long; femur 10 (8-10) long, ventral basifemoral seta $b v 12$ (7-12) long; genu 5 (5-6) 


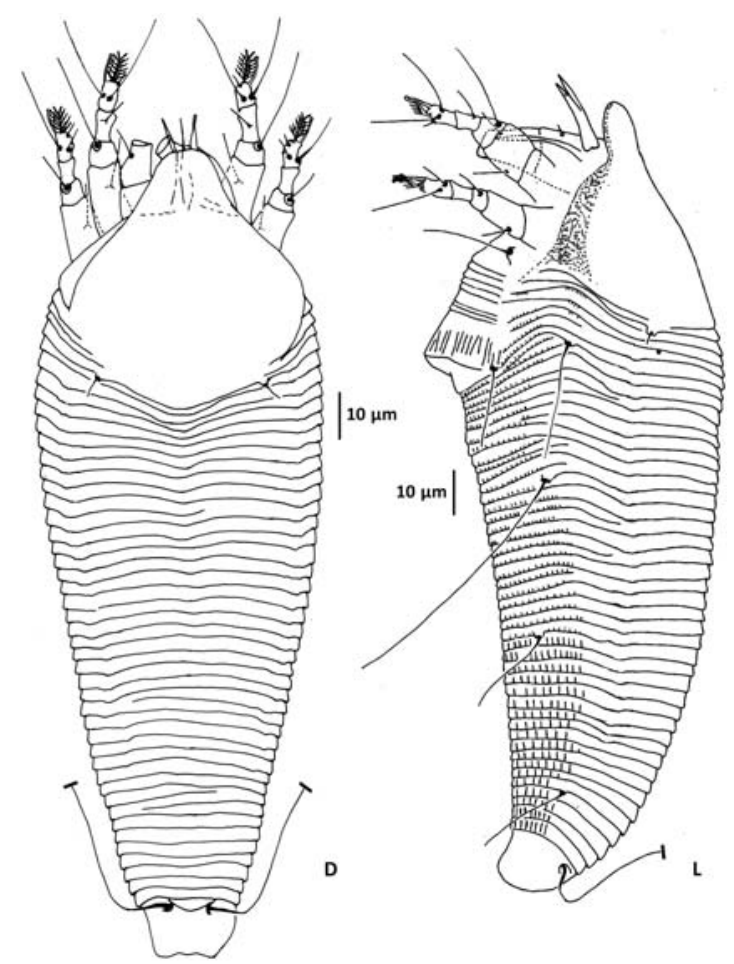

Fig. 2. Abacarus asperi (Chandrapatya \& Boczek)

long, antaxial genual seta $l^{\prime \prime} 22$ (22-30) long; tibia 6 (6-8) long, paraxial tibial seta $l^{\prime} 9$ (6-11) long; tarsus 8 (6-8) long, antaxial fastigial tarsal seta $f t^{\prime \prime} 20$ (17-25) long, paraxial fastigial tarsal seta $f t^{\prime} 19$ (17-22) long, paraxial unguinal tarsal seta $u^{\prime} 5$ (4-5) long, solenidion slightly curved, knobbed, 6 (6-7) long; empodium simple, 6 (5-6) long, 6-rayed. Leg II 27 (25-27) long; femur 9 (7-9) long, ventral basifemoral seta $b v 8$ (8-11) long; genu 4 (4-6) long, antaxial genual seta $l$ " 8 (8-10) long; tibia 5 (4-5) long; tarsus 6 (5-7) long, antaxial fastigial tarsal seta $f t^{\prime \prime} 22$ (20-25) long, paraxial fastigial tarsal seta $f t^{\prime} 7$ (6-7) long, paraxial unguinal tarsal seta $u^{\prime} 4$ (3-5) long, solenidion as in leg I, 7 (5-7) long; empodium simple, 6 (4-6) long, 6-rayed. Coxisternal plates: with a few faint curved lines on both coxisterna; coxisternum I not touching mesally, forming 8 (8-10) long longitudinal narrow sternal groove. Anterior seta on coxisternum I $1 b 8$ (4-8) long, 10 (9-12) apart; proximal seta on coxisternum I la 20 (20-25) long, 9 (8-9) apart; proximal seta on coxisternum II $2 a 22$ (22-45) long, 17 (17-23) apart; distance between setae $1 b$ and $1 a 9$ (79) long; coxigenital semiannuli 8 (6-8), smooth. Opisthosoma: with 43 (41-46) smooth dorsal annuli. Ventral opisthosoma with 52 (47-56) annuli, with short microtubercles situated on rear border of annuli, microtubercles posterior to opisthosomal seta $e$ toward the end of the body more elongate. First 33-39 dorsal annuli forming 2 subdorsal ridges and only 19-21 annuli forming indistinct middorsal ridge. Opisthosomal seta $c 225$ (25-30) long, 50 (49-59) apart, on annulus 8 (7-9); opisthosomal seta $d 56$ (45-60) long, 30 (28-32) apart, on annulus 21 (18-22); 

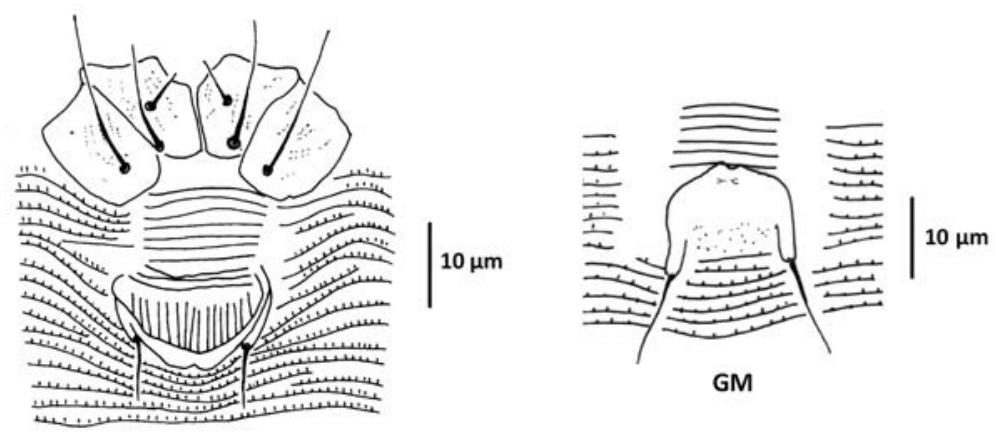

GF
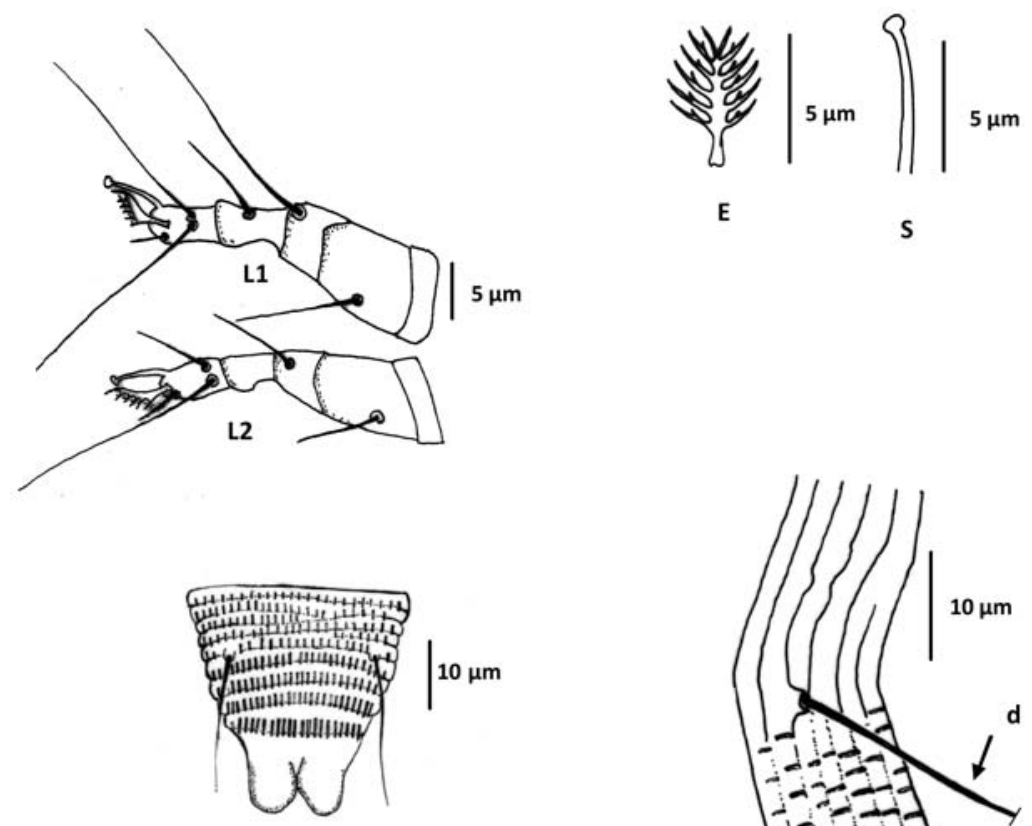

CS

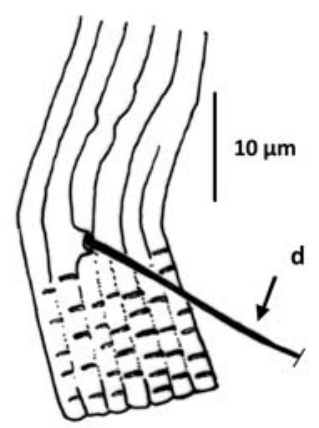

LO

Fig. 2. (Continue) Abacarus asperi (Chandrapatya \& Boczek)

opisthosomal seta e 20 (15-20) long, 17 (15-18) apart, on annulus 34 (28-36); opisthosomal seta $f 20$ (18-25) long, 24 (23-25) apart, on annulus 48 (42-51), or 5 from the rear. Opisthosomal seta $h 235$ (35-45) long; opisthosomal seta $h l$ absent. Genital coverflap: 21 (21-28) wide, 15 (12-15) long, with three transverse lines basally and 16 (14-18) longitudinal ridges distally; proximal seta on coxisternum III $3 a 20$ (10-20) long, 13 (13-16) apart.

MALE $(\mathrm{n}=2)$. Smaller than female, 146-190 long, 55 wide. Gnathosoma: 24 long; pedipalp coxal seta ep 2 long, dorsal pedipalp genual seta $d$ 4-5 long. Chelicerae 16-18 long. Prodorsal 
shield: 46-57 long, 47-52 wide, similar to female. Scapular setae sc 3-4 long, on scapular tubercles 30-35 apart. Prodorsal shield frontal lobe 10 long. Legs: leg I 23-25 long; femur 8 long, ventral basifemoral seta $b v$ 7-10 long; genu 4-5 long, antaxial genual seta $l$ "16-28 long; tibia 5-6 long, paraxial tibial seta $l^{\prime} 8-10$ long; tarsus 5-6 long, antaxial fastigial tarsal seta $\mathrm{ft}^{\prime \prime}$ 16-20 long, paraxial fastigial tarsal seta $f t^{\prime} 14-20$ long, paraxial unguinal tarsal seta $u^{\prime}$ 4-5 long, solenidion slightly curved, knobbed, 5-6 long; empodium simple, 5-6 long, 6-rayed. Leg II 21-25 long; femur 7-8 long, ventral basifemoral seta $b v$ 6-10 long; genu 4-5 long, antaxial genual seta $l$ " 6-10 long; tibia 4-5 long; tarsus 5-7 long, antaxial fastigial tarsal seta $f t^{\prime \prime}$ 15-22 long, paraxial fastigial seta $f t^{\prime}$ 5-6 long, paraxial unguinal seta $u^{\prime} 4$ long, solenidion slightly curved, knobbed, 5-6 long; empodium simple, 5 long, 6-rayed. Coxisternal plates: with a few faint longitudinal curved lines on both coxisterna. Internal coxisternal apodeme a groove 8 long. Anterior seta on coxisternum I $1 b$ 5-6 long, 10-12 apart; proximal seta on coxisternum I 1a 20-23 long, 7-9 apart; proximal seta on coxisternum II $2 a$ 25-27 long, 20-22 apart; distance between setae $1 b$ and $1 a$ 6-7 long; coxigenital semiannuli 6-7, smooth. Opisthosoma: with 46-47 smooth dorsal annuli, 54 microtuberculate ventral annuli. Opisthosomal seta $c 230$ long, 43-50 apart, on annulus 8-9; opisthosomal seta $d 42-50$ long, 23-28 apart, on annulus 20; opisthosomal seta $e$ 15-17 long, 1115 apart, on annulus 33-35; opisthosomal seta $f$ 16-18 long, 18-24 apart, on annulus 46 (42-50), or 5 from the rear. Opisthosomal seta $h 2$ 35-38 long; opisthosomal seta $h l$ absent. Genitalia: 1719 wide, proximal seta on coxisternum III $3 a$ 13-15 long, 15-16 apart.

Etymology: the species name is based on the host plant species.

Type material: female holotype on slide \#600.1; 20 female and male paratypes on slides labeled \#600.1.

Host plant and locality: collected from leaves of Dendrocalamus asper (Schult. \& Schult. f.) Backer ex K. Heyne (Phai tong), Poaceae, Bambuseae, Amphoe Khlung, Chanthaburi Province, Thailand, 3 December 1997, collected by A. Chandrapatya.

Other hosts and distribution: Dendrocalamus asper (Schult. \& Schult. f.) Backer ex K. Heyne (Phai tong), Poaceae, Bambuseae, Amphoe Si Racha, Chon Buri Province, Thailand, 13 October 1998, collected by A. Chandrapatya (38 slides labeled \#952.1).

Relation to host: vagrant on both leaf surfaces. No apparent damage was observed.

Remarks: Bamboocarus Chandrapatya and Boczek, 2002 was made a junior synonym of Aculus Keifer 1959 by Amrine et al. (2003) but the opisthosoma of this mite has a trough with 3 ridges, the middorsal ridge ending before subdorsal ridges. It is moved to Abacarus, new combination.

Abacarus klaengensis, replacement name for Abacarus bambusae (Boczek, 1998), new combination, junior homonym of Abacarus bambusae Kuang and Zhuo, 1987. The mite described as Phytoptochetus bambusae Boczek, 1998, belongs in Abacarus. Because it becomes a junior homonym of Abacarus bambusae Kuang \& Zhuo, 1987, it needs a new name, provided here as klaengensis.

Phytoptochetus bambusae Boczek, 1998; Boczek \& Chandrapatya, 1998: 138-139, f.3.

Abacarus bambusae (Boczek, 1998), new combination. 
Abacarus bambusae Kuang \& Zhuo, 1987: 380-382, senior homonym.

\section{Redescription:}

FEMALE (Fig.3) (n=14). Body slightly fusiform, 150 (112-150) long, 49 (40-52) wide, (4654) thick; color in life light red. Gnathosoma: projecting slightly downwards, 22 (20-23) long; pedipalp coxal seta ep 3 long, dorsal pedipalp genual seta $d 5$ (4-6) long. Chelicerae 17 (1722) long, almost straight. Prodorsal shield: 35 (35-42) long, 41 (35-45) wide, subtriangular, posteriorly elongate, ornamented with numerous granules. Scapular setae sc 14 (12-20) long on scapular tubercles 24 (22-26) apart, situated on rear shield margin, directed divergently to the rear. Shield design of median, admedian, submedian and three oblique lateral lines on each side; antero-lateral region and between some lines, ornamented with numerous granules. Prodorsal shield frontal lobe triangular, anteriorly rounded, extending over gnathosoma, 4 (3-5) long. Legs: with all setae present. Leg I 30 (27-33) long; femur 11 (10-12) long, ventral basifemoral seta $b v 12$ (8-15) long; genu 5 (4-5) long, antaxial genual seta $l$ " 32 (30-35) long; tibia 6 (5-7) long, paraxial tibial seta $l^{\prime} 8$ (5-10) long; tarsus 8 (6-8) long, antaxial fastigial tarsal seta $f t^{\prime \prime} 24$ (18-24) long, paraxial fastigial tarsal seta $f t^{\prime} 23$ (16-23) long, paraxial unguinal tarsal seta $u^{\prime} 4$ (4-7) long, solenidion slightly curved, tapered apically, 10 (7-10) long; empodium simple, 10 (8-10) long, 7-rayed. Leg II 28 (23-29) long; femur 11 (7-11) long, ventral basifemoral seta $b v$ 11 (11-22) long; genu 5 (3-5) long, antaxial genual seta $l^{\prime \prime} 18$ (10-18); tibia 5 (5-6) long; tarsus 7 (6-7) long, antaxial fastigial tarsal seta $f t^{\prime \prime} 21$ (20-25) long, paraxial fastigial tarsal seta $f t^{\prime} 11$ (6-11) long, paraxial unguinal tarsal seta $u^{\prime} 4$ (4-5) long, solenidion as in leg I, 8 (7-10) long; empodium simple, 9 (6-11) long, 7-rayed. Coxisternal plates: with numerous short lines on both coxisterna. Internal coxisternal apodeme a line 8 (8-15) long. Anterior seta on coxisternum I $1 b$ 8 (6-10) long, 13 (8-13) apart; proximal seta on coxisternum I la 21 (15-25) long, 7 (6-10) apart; proximal seta on coxisternum II $2 a 31$ (27-42) long, 23 (17-23) apart; distance between setae $1 b$ and $1 a 7$ (5-7) long; coxigenital semiannuli 5 (5-6), microtuberculate. Opisthosoma: with 51 (43-52) dorsal annuli, microtubercles slightly pointed, situated behind rear border of annuli. Ventral opisthosoma with 55 (53-65) annuli, with fine microtubercles situated on rear margin of annuli. Microtubercles elongated on the last 9-11 ventral annuli. Dorsal annuli forming three longitudinal ridges. Middorsal opisthosomal ridge extending from shield to annuli 32-41; subdorsal ridges extending from shield to annuli 40-46. Opisthosomal seta $c 232$ (20-32) long, 45 (35-45) apart, on annulus 8 (8-10); opisthosomal seta $d 50$ (50-70) long, 28 (25-32) apart, on annulus 18 (16-22); opisthosomal seta $e 30$ (30-45) long, 16 (14-18) apart, on annulus 31 (30-38); opisthosomal seta $f 22$ (20-25) long, 18 (17-20) apart, on annulus 52 (48-59), or 5 (5-6) from the rear. Opisthosomal seta $h 253$ (46-55) long; opisthosomal seta $h 11$ long. Genital coverflap: 20 (15-21) wide, 15 (12-15) long, with 10-12 longitudinal ridges; proximal seta on coxisternal plate III $3 a 15$ (15-18) long, 17 (13-17) apart.

MALE $(\mathrm{n}=2)$. Smaller than female, 128-129 long, 45 wide, 45 thick. Gnathosoma: 19 long; pedipalp coxal seta ep 3 long, dorsal pedipalp genual seta $d 4-6$ long. Chelicerae 18 long. Prodorsal shield: 32-38 long, 41-42 wide, similar to female. Scapular setae sc 12-15 long, on scapular tubercles 21-23 apart. Prodorsal shield frontal lobe 3-4 long. Legs: leg I 2527 long; femur 9-10 long, ventral basifemoral seta $b v 10$ long; genu 4-5 long, antaxial genual seta $l^{\prime \prime} 20$ long; tibia 6-7 long, paraxial tibial seta $l^{\prime} 6$ long; tarsus 5 long, antaxial fastigial tarsal 


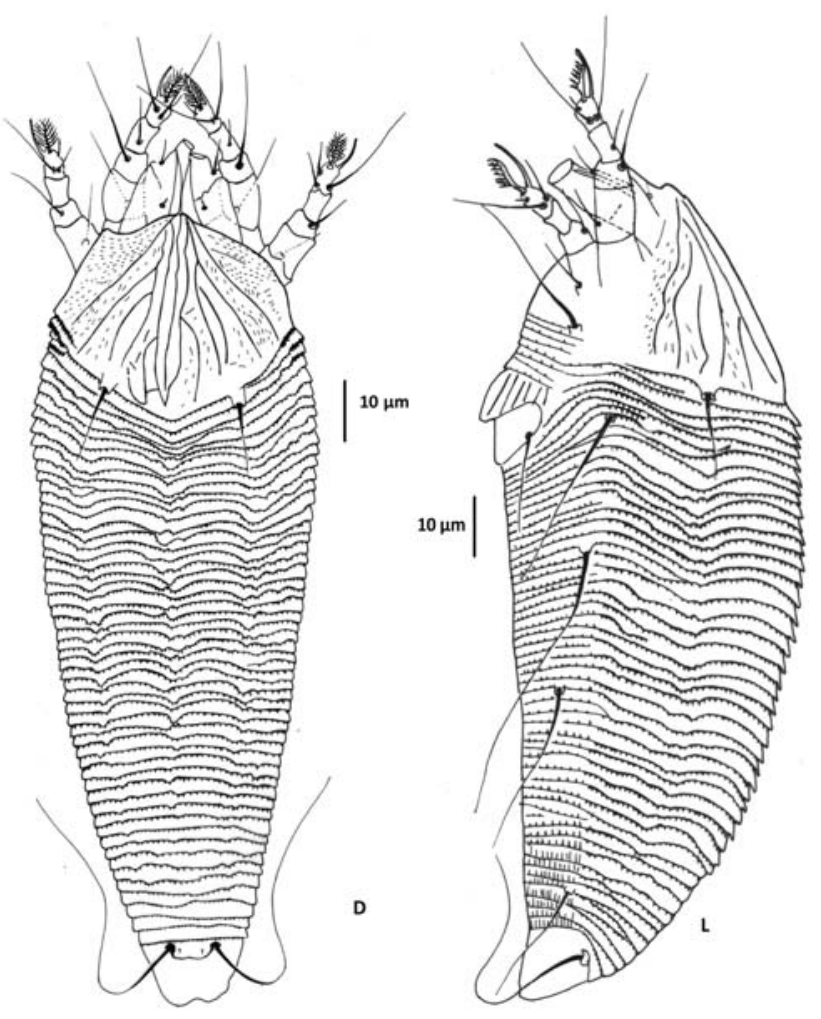

Fig. 3. Abacarus klaengensis (Boczek)

seta $f t^{\prime \prime} 20$ long, paraxial fastigial tarsal seta $f t^{\prime} 20$ long, paraxial unguinal tarsal seta $u^{\prime} 5$ long, solenidion slightly curved, tapered apically, 8-9 long; empodium simple 7-8 long, 7-rayed. Leg II 23-26 long; femur 8-10 long, ventral basifemoral bv 20 long; genu 4-5 long, antaxial genual seta $l^{\prime \prime} 11-14$ long; tibia 5 long; tarsus 6 long, antaxial fastigial tarsal seta $f t^{\prime \prime} 20$ long, paraxial fastigial tarsal seta $f t^{\prime}$ 7-8 long, paraxial unguinal tarsal seta $u^{\prime}$ 4-5 long, solenidion slightly curved, tapered apically, 8-9 long; empodium simple, 7-8 long, 7-rayed. Coxisternal plates: with numerous short lines on both coxisterna. Internal coxisternal apodeme a line 8-9 long. Anterior seta on coxisternum I $1 b 8$ long, 10-11 apart; proximal seta on coxisternum I la 13-24 long, 8 apart; proximal seta on coxisternum II $2 a$ 34-40 long, 17-19 apart; distance between setae $1 b$ and 1a 6-7 long; coxigenital semiannuli 6-7, microtuberculate. Opisthosoma: with 44-45 dorsal annuli, 52 ventral annuli, both dorsal and ventral annuli microtuberculate. Opisthosomal seta $c 2$ 25-35 long, 36-37 apart, on annulus 9; opisthosmal seta $d$ 40-50 long, 24-25 apart, on annulus 18-19; opisthosomal seta $e$ 32-35 long, 13-14 apart, on annulus 30-31; opisthosomal seta $f$ 2025 long, 18 apart, on annulus 46 (46-47), or 5-6 from the rear. Opisthosomal seta $h 245$ long; opisthosomal seta $h l 1$ long. Genitalia: 16-18 wide, proximal seta on coxisternum III $3 a$ 12-14 long, 12-13 apart.

Etymology: the species name is based on the name of the location (Amphoe Klaeng) where 

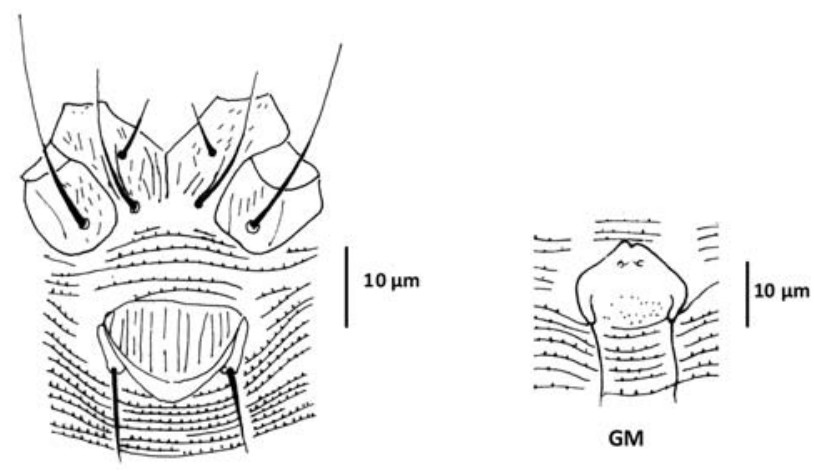

GM

GF
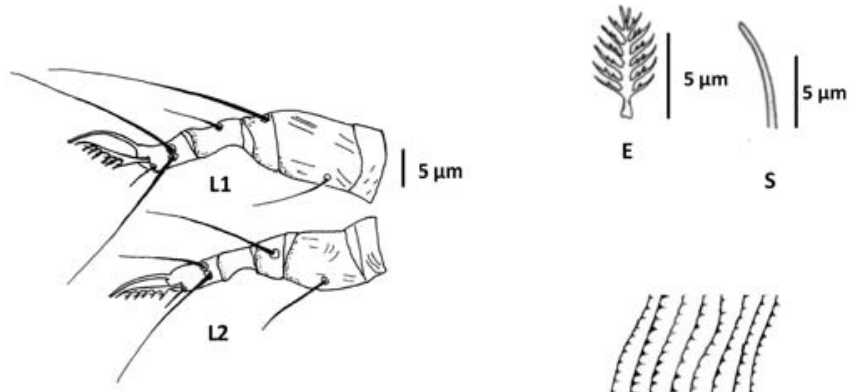

S

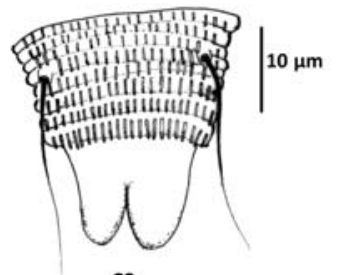

CS

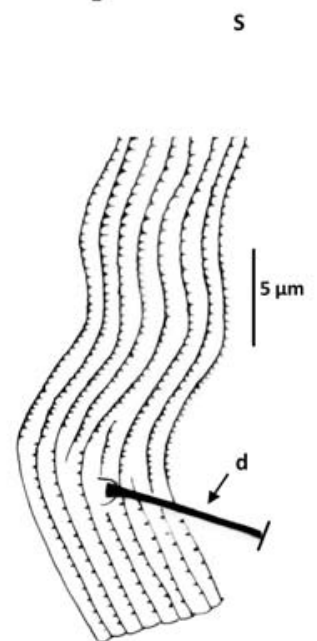

LO

Fig. 3. (Continue) Abacarus klaengensis (Boczek)

the mite was collected.

Type material: female and male paratypes on 10 slides labeled \#191 and 21 slides labeled \#517.

Host plant and locality: collected from leaves of Bambusa sp. (Phai), Poaceae, Bambuseae, Ban Pae, Amphoe Klaeng, Rayong Province, Thailand, 2 November 1996, collected by A. Chandrapatya; and from Bambusa sp. (Phai), Poaceae, Bambuseae, Mahidol University, Khet Phaya Thai, Bangkok, Thailand, 5 August 1997, collected by A. Chandrapatya. 
Other hosts and distribution: Bambusa multiplex (Lour.) Raeusch. ex Schult. \& Schult. f. (Phai liang), Poaceae, Bambuseae, Mahidol University, Khet Phaya Thai, Bangkok, Thailand, 14 October 1998, collected by A. Chandrapatya (7 slides labeled \#987); Bambusa multiplex (Lour.) Raeusch. ex Schult. \& Schult. f. (Phai liang), Poaceae, Bambuseae, Amphoe Kamphaeng Saen, Nakhon Pathom Province, Thailand, 29 April 1999, collected by A. Chandrapatya (20 slides labeled \#1420.1).

Relation to host: vagrant on the lower leaf surface. No apparent damage was observed.

Diagnosis: Abacarus klaengensis is close to Abacarus sacchari ChannaBasavanna, Abacarus delhiensis ChannaBasavanna and Abacarus oryzae Keifer, 1963, but differs by shield design and structure of the dorsal opisthosoma. Median line on prodorsal shield of A. sacchari is absent, two admedian lines are complete, middle part of the shield smooth with numerous granules laterally. Shield design of A. delhiensis has distinctive granules with complete median and admedian lines. In contrast, the shield of A. klaengensis has median, admedian, submedian and three oblique lateral lines, with the antero-lateral region and between some lines ornamented with numerous granules. The shield of A. oryzae has median, admedian and two submedian lines; the admedian lines converge posterior to median line, unlike klaengensis. Dorsal opisthosoma of A. klaengensis is broader than A. oryzae; both A. sacchari and A. delhiensis have a wider and shorter middorsal ridge, respectively and the dorsal opisthosoma of both are smooth.

\section{Knorella blumeanae Xue \& Zhang, 2009: 50; Emendation.}

Knorella blumcanae Xue \& Zhang, 2009: 50; (authors named the mite after Bambusa blumeana and misspelled the species designation), replacement name for Knorella bambusae (Chandrapatya, 1998).

Thiracarus bambusae Chandrapatya, 1998; Boczek \& Chandrapatya, 1998: 136-138, f. 2. The genus Thiracarus is a junior synonym of Knorella Keifer, 1975 (Amrine et al., 2003).

Knorella bambusae (Chandrapatya, 1998); Amrine et al., 2003: 59,

a junior homonym of Knorella bambusae (Kuang \& Feng, 1989) which was reassigned to Knorella from Neoknorella bambusae Kuang \& Feng, 1989 (Kuang \& Feng, 1989: 37-41) by Amrine \& Stasny, 1994: 222; this mite is distinctly different from Knorella bambusae (Kuang $\&$ Feng, 1989), thus it needed a new name. This is another example of the extensive confusion resulting from 24 mites named 'bambusae', especially when mites are reexamined and found to be assigned to incorrect genera.

\section{Redescription:}

FEMALE (Fig.4) (n=10). Body fusiform, 148 (125-148) long, 58 (51-58) wide, 49 (49-55) thick; color in life light yellow. Gnathosoma: projecting downwards, 20 (18-25) long; pedipalp coxal seta ep 2 (2-3) long, dorsal pedipalp genual seta $d$ absent. Chelicerae 19 (14-22) long, almost straight. Prodorsal shield: 44 (40-47) long, 58 (51-58) wide, semicircular. Scapular setae $s c$ and scapular tubercles absent. Shield design consists of 2 admedian lines very close to each other, laterally with several granules. Prodorsal shield frontal lobe small, broad-based, extending over gnathosoma, 2 (2-5) long. Legs: lacking setae on femur I, femur II and genu II. Leg I 22 (22-27) long; femur 8 (8-10) long, with numerous granules on the surface, ventral basifemoral seta $b v$ absent; genu 4 (3-5) long, antaxial genual seta $l$ " 22 (20-25) long; tibia 3 (3-4) long, 

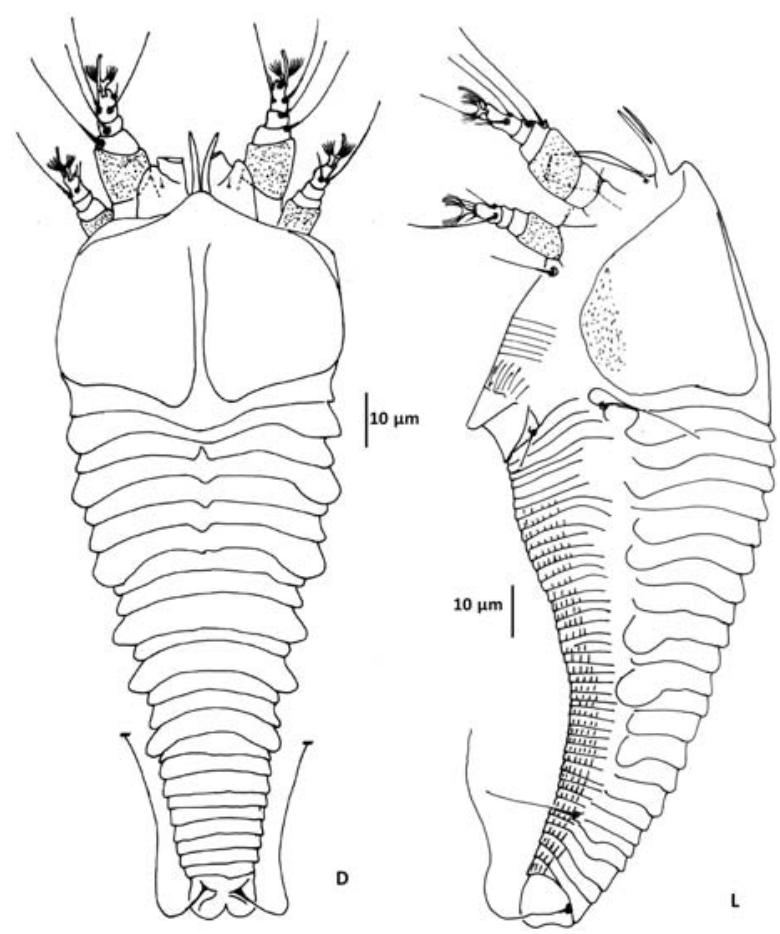

Fig. 4. Knorella blumeanae (Chandrapatya)

paraxial tibial seta $l^{\prime} 19$ (18-23) long; tarsus 7 (5-7) long, antaxial fastigial tarsal seta $f t^{\prime \prime} 15$ (1522) long, paraxial fastigial tarsal seta $f t^{\prime} 15$ (10-18) long, paraxial unguinal tarsal seta $u^{\prime} 3$ (3-5) long, solenidion slightly curved, tapered apically, 6 (6-8) long; empodium divided, 6 (4-8) long 6-7 rayed. Leg II 18 (17-22) long; femur 8 (7-10) long, with granules on the surface, ventral basifemoral seta $b v$ absent; genu 2 (2-4) long, antaxial genual seta $l$ " absent; tibia 2 (2-3) long; tarsus 6 (5-7) long, antaxial fastigial tarsal seta $f t^{\prime \prime} 15$ (15-20) long, paraxial fastigial tarsal seta $\mathrm{ft}^{\prime}$ 8 (6-8) long, paraxial unguinal tarsal seta $u^{\prime} 3$ (3-5) long, solenidion almost straight, 6 (5-7) long; empodium 6 (4-6) long, divided, 6-7 rayed. Coxisternal plates: with short lines and granules on both coxisterna. Internal coxisternal apodeme a line 7 (7-14) long. Anterior seta on coxisternum I $1 b 7$ (5-8) long, 14 (10-14) apart; proximal seta on coxisternum I $1 a 15$ (15-25) long, 8 (5-9) apart; proximal seta on coxisternum II $2 a 22$ (15-25) long, 22 (17-22) apart; distance between setae $1 b$ and $1 a 12$ (10-12) long; coxigenital semiannuli 4 (4-6), smooth. Opisthosoma with 24 (23-25) smooth dorsal annuli. Ventral opisthosoma with 47 (47-53) annuli, with elongate microtubercles situated on rear margin of annuli. Opisthosoma with a short middorsal ridge; annuli 2, 4, 7, 10, 12 and 15 forming distinct lateral lobes. Microtubercles elongated on the last ventral annulus. Opisthosomal seta $c 220$ (14-30) long, 39 (36-42) apart, on annulus 7 (67); opisthosomal setae $d$ and $e$ absent; opisthosomal seta $f 20$ (17-25) long, 17 (16-20) apart, on annulus 40 (40-47), or 6 from the rear. Opisthosomal seta $h 260$ (35-60) long; opisthosomal seta hl absent. Genital coverflap: 19 (16-20) wide, 18 (14-18) long, with 14-15 longitudinal ridges; 

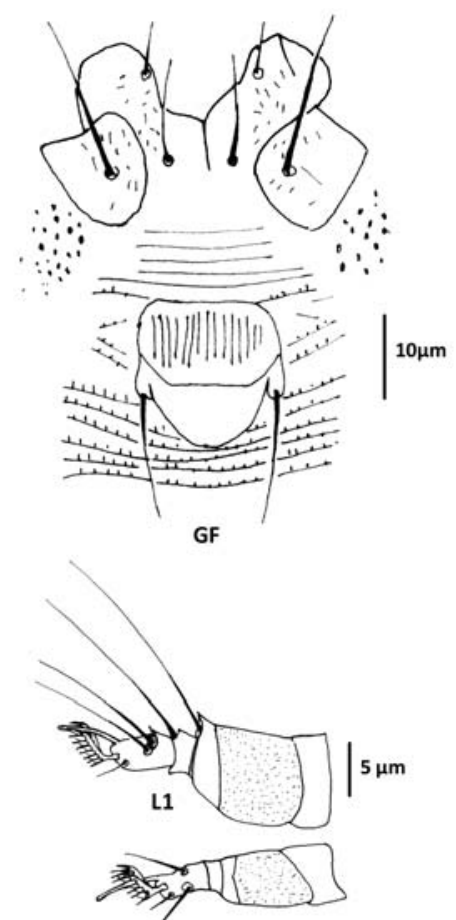

L2

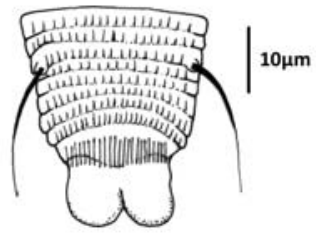

CS
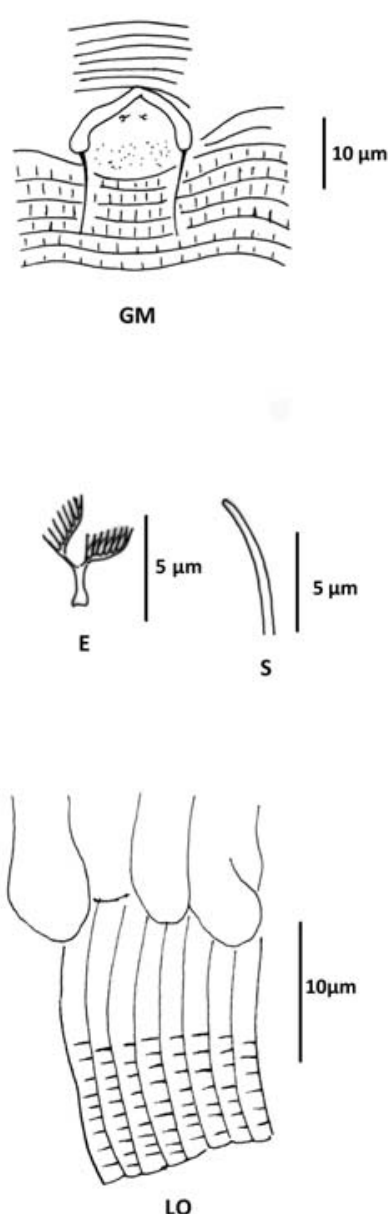

Fig. 4. (Continue) Knorella blumeanae (Chandrapatya)

proximal seta on coxisternum III $3 a 16$ (10-16) long, 17 (13-17) apart.

MALE $(\mathrm{n}=3)$. 126-128 long, 48-53 wide, 50-51 thick. Gnathosoma: 18-24 long; pedipalp coxal seta ep 2-3 long, dorsal pedipalp genual seta $d$ absent. Chelicerae 16-18 long. Prodorsal shield: 38-40 long, 47-53 wide, similar to female. Scapular setae sc and scapular tubercles absent. Prodorsal shield frontal lobe 2-6 long. Legs: leg I 19-23 long; femur 8 long, ventral basifemoral seta $b v$ absent; genu 3-4 long, antaxial genual seta $l$ " 20-22 long; tibia 3-4 long, paraxial tibial seta $l^{\prime}$ 15-20 long; tarsus 5-6 long, antaxial fastigial tarsal seta $f t^{\prime \prime}$ 18-20 long, paraxial fastigial tarsal seta $f t^{\prime} 15$ long, paraxial unguinal tarsal seta $u^{\prime}$ 4-5 long, solenidion slightly curved, tapered apically, 6 long; empodium divided, 5 long, 6-7 rayed. Leg II 17-20 
long; femur 8-10 long, ventral basifemoral seta $b v$ absent; genu 2-3 long, antaxial genual seta $l^{\prime \prime}$ absent; tibia 2-3 long; tarsus 5-6 long, antaxial fastigial tarsal seta $\mathrm{ft}^{\prime \prime}$ 17-20 long, paraxial fastigial tarsal seta $f t^{\prime}$ 6-8 long, paraxial unguinal tarsal seta $u^{\prime}$ 4-5 long, solenidion almost straight, tapered apically, 5 long; empodium divided, 4 long, 6-7 rayed. Coxisternal plates: with granules and short lines on both coxisterna. Internal coxisternal apodeme a line 7-8 long. Anterior seta on coxisternum I $1 b$ 5-8 long, 11 apart; proximal seta on coxisternum I 1a 15-18 long, 5-8 apart; proximal seta on coxisternum II 2a 15-20 long, 15-22 apart; distance between setae $1 b$ and $1 a 10$ long; coxigenital semiannuli 6-8, smooth. Opisthosoma: with 24 smooth dorsal annuli, 49-54 microtuberculate ventral annuli. Opisthosomal seta $c 2$ 15-30 long, 32-40 apart, on annulus 7; opisthosomal setae $d$ and $e$ absent; opisthosomal seta $f$ 15-25 long, 16-18 apart, on annulus 48 (43-48), or 6 from the rear. Opisthosomal seta $h 2$ 30-35 long; opisthosomal seta $h l$ absent. Genitalia: 13-14 wide, proximal seta on coxisternum III $3 a$ 13-15 long, 12 apart.

Etymology: the species was named after the host species name, blumeana.

Type material: female and male paratypes on 6 slides labeled \#160 and 35 slides labeled \#230.

Host plant and locality: collected from leaves of Bambusa blumeana Schult. f. (Phai si suk), Poaceae, Bambuseae, Kasetsart University, Bangkhen Campus, Khet Chatuchak, Thailand, 1 November 1996, collected by A. Chandrapatya; and from Bambusa blumeana (Phai si suk), Poaceae, Bambuseae, Amphoe Sam Phran, Nakhon Pathom Province, Thailand, 2 November 1996, collected by A. Chandrapatya.

Other hosts and distribution: Bambusa multiplex (Lour.) Raeusch. ex Schult. \& Schult. f. (Phai liang), Poaceae, Bambuseae, Amphoe Sam Phran, Nakhon Pathom Province, Thailand, 2 November 1996, collected by A. Chandrapatya (20 slides labeled \#228); Bambusa sp. (Phai), Poaceae, Bambuseae, Mahidol University, Khet Phaya Thai, Bangkok, Thailand, 5 August 1997, collected by A. Chandrapatya (three slides labeled \# 517.1); Bambusa sp. (Phai), Poaceae, Bambuseae, Amphoe Si Racha, Chon Buri Province, Thailand, 13 September 1998, collected by A. Chandrapatya (38 slides labeled \#948).

Relation to host: vagrant on the lower leaf surface. No apparent damage was observed.

Remarks: Amrine et al. (2003) placed Thiracarus Chandrapatya, 1998 as a junior synonym of Knorella Keifer, 1975; Knorella bambusae Kuang \& Feng, 1989 thus became the senior homonym. Xue \& Zhang (2009) designated 'blumcana' as the species designation for this mite, but the host species name is blumeana, hence, the mite name is emended here to Knorella blumeanae (Xue \& Zhang, 2009).

\section{Knorella thailandica Hong, 1996}

This is a replacement name for Knorella bambusae Chandrapatya, 1993 (Hong, 1996). Chandrapatya \& Boczek, 1993: 45-47, f.1, junior homonym of Knorella bambusae (Kuang \& Feng, 1989); Kuang \& Feng, 1989. Description of a new genus and three new species of Phyllocoptinae from China (Acariformes: Eriophyoidea). Acta Zootax. Sin., 14(1): 37-41(As Neoknorella bambusae Kuang \& Feng, 1989).

\section{Redescription:}

FEMALE (Fig.5) ( $\mathrm{n}=13$ ). Body cone-shaped, much broader anteriorly, 114 (104-117) long, 50 (45-53) wide, (41-50) thick; color in life yellow. Gnathosoma: projecting downwards, 17 (17- 

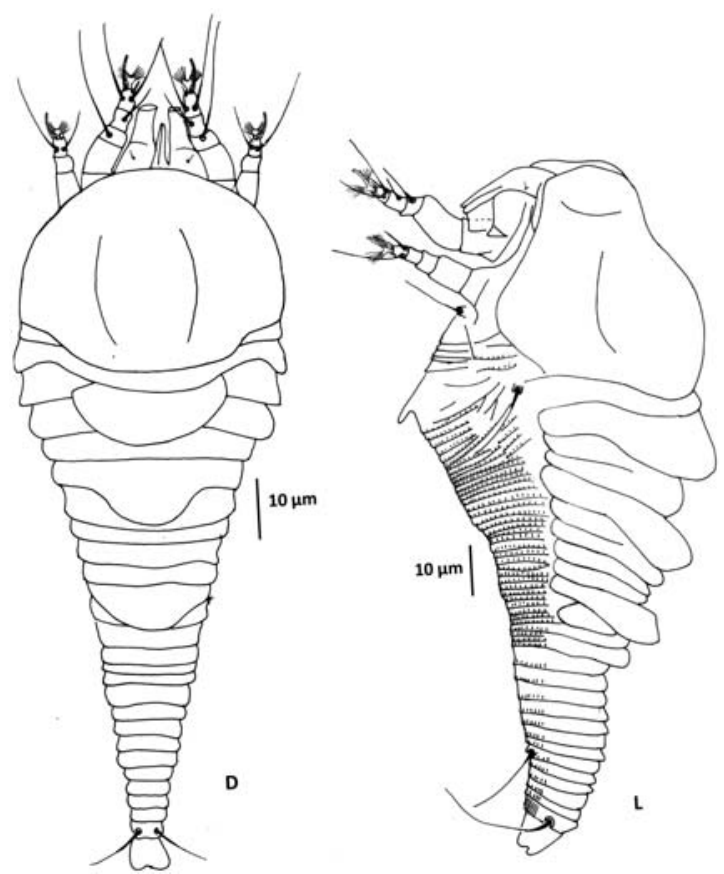

Fig. 5. Knorella thailandica (Chandrapatya) Hong

20) long; pedipalp coxal seta ep 2 (2-3) long, dorsal pedipalp genual seta $d$ absent. Chelicerae 16 (16-20) long, almost straight. Prodorsal shield: 37 (31-42) long, 50 (45-53) wide, semicircular. Scapular setae $s c$ and tubercles absent. Shield design of two admedian lines and a transverse, curved line antero-laterally. Prodorsal shield frontal lobe rounded, oriented vertically, small, broad-based, extending over gnathosoma, 2 (1-3) long. Legs: lacking setae on femur I, femur II and genu II. Leg I 20 (20-23) long; femur 9 (6-9) long, ventral basifemoral seta $b v$ absent; genu 3 (3-4) long, antaxial genual seta $l^{\prime \prime} 19$ (12-18) long; tibia 3 (2-3) long, paraxial tibial seta $l^{\prime} 12$ (12-16) long; tarsus 5 (5-6) long, antaxial fastigial tarsal seta $f t^{\prime \prime} 17$ (14-20) long, paraxial fastigial tarsal seta $f t^{\prime} 16$ (13-16) long, paraxial unguinal tarsal seta $u^{\prime} 3$ (3-5) long, solenidion slightly curved, tapered apically, 6 (5-6) long; empodium divided, 4 (4-5) long, 6-rayed. Leg II 21 (16-21) long; femur 9 (6-9) long, ventral basifemoral seta $b v$ absent; genu 3 (2-3) long, antaxial genual seta $l$ " absent; tibia 3 (2-3) long; tarsus 4 (4-5) long, antaxial fastigial tarsal seta $f t^{\prime \prime} 15$ (14-20) long, paraxial fastigial tarsal seta $f t^{\prime} 7$ (6-9) long, paraxial unguinal tarsal seta $u^{\prime} 3$ (3-6) long, solenidion as in leg I, 5 (5-6) long; empodium 4 (4-5) long, divided, 6-rayed. Coxisternal plates: both coxisterna with smooth surfaces. Coxisternum I fused, not forming internal coxisternal apodeme. Anterior seta on coxisternum I $1 b 8$ (6-9) long, situated on strong tubercle, at the middle part of coxisternum 15 (14-15) apart; proximal seta on coxisternum I $1 a$ 12 (10-15) long, 8 (6-8) apart; proximal seta on coxisternum II $2 a 15$ (15-20) long, 23 (19-30) apart; distance between setae $1 b$ and $l a 8$ (6-8) long; coxigenital semiannuli 4 (4-5), smooth. Opisthosoma: with 24 (22-25) smooth dorsal annuli. Annuli 1, 2 or 3, 5 or 6 and 10 or 11 

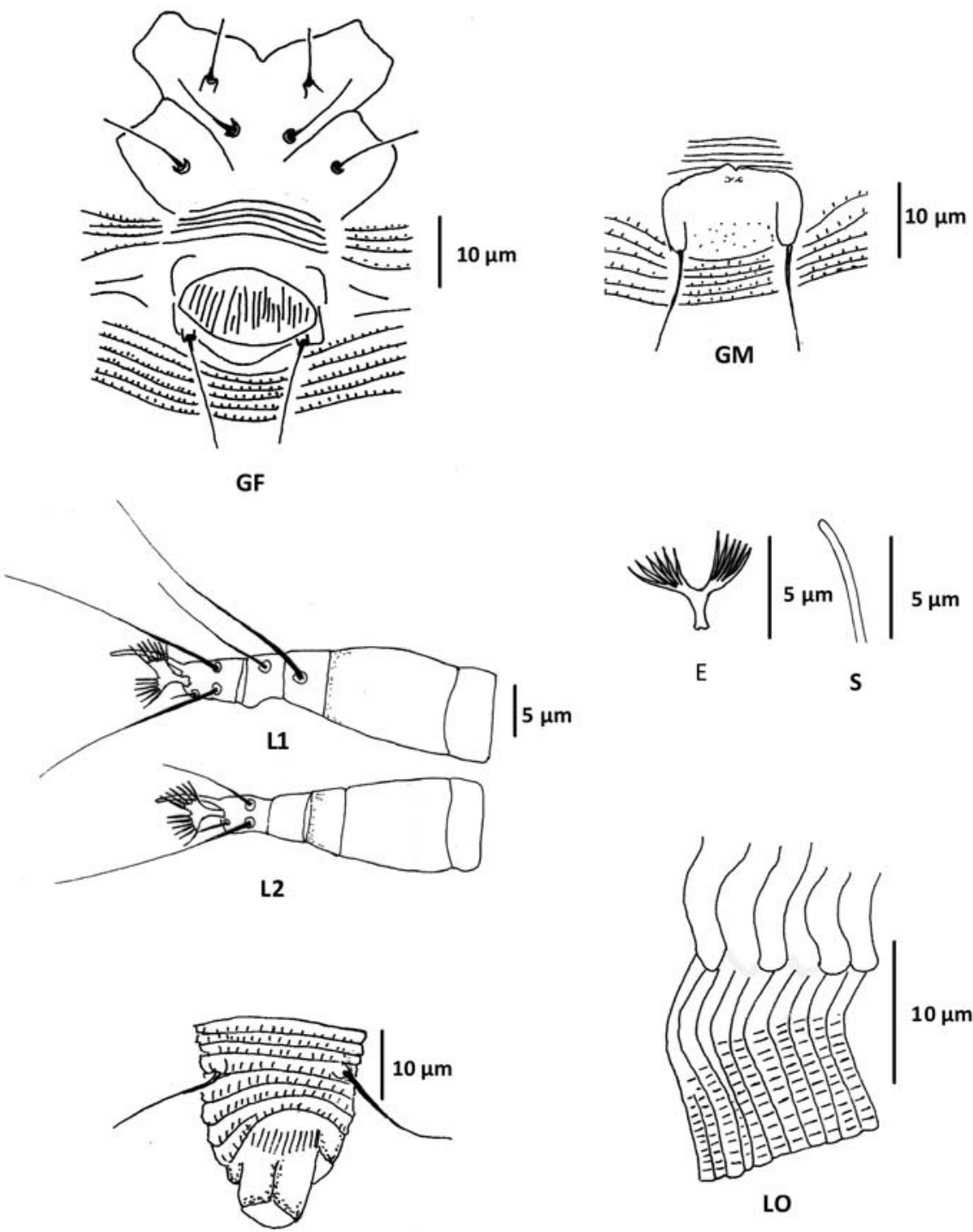

CS

Fig. 5. (Continue) Knorella thailandica (Chandrapatya) Hong

forming broad, median lobes overhanging next 1-2 annuli. Ventral opisthosoma with 55 (44-55) annuli, with elongate microtubercles situated on rear margin of annuli. Microtubercles elongated on the last annulus. Opisthosomal seta $c 216$ (15-20) long, 39 (32-39) apart, on annulus 5 (46); opisthosomal seta $d$ and $e$ absent; opisthosomal seta $f 16$ (16-20) long, 14 (13-15) apart, on annulus 49 (44-49), or 6 (6-7) from the rear. Opisthosomal seta $h 232$ (23-40) long; opisthosomal seta $h l$ absent. Genital coverflap: 20 (17-20) wide, 12 (10-14) long, with numerous longitudinal 
ridges; proximal seta on coxisternum III $3 a 17$ (13-17) long, 17 (11-17) apart.

MALE $(\mathrm{n}=1)$. 116 long, 50 wide, 42 thick. Gnathosoma: 19 long; pedipalp coxal seta $e p$ 2 long, dorsal pedipalp genual seta $d$ absent. Chelicerae 17 long. Prodorsal shield: 40 long, 50 wide, similar to female. Scapular setae $s c$ and tubercles absent. Prodorsal shield frontal lobe 2 long. Legs: leg I 19 long; femur 8 long, ventral basifemoral seta $b v$ absent; genu 3 long, antaxial genual seta $l^{\prime \prime} 20$ long; tibia 3 long, paraxial tibial seta $l^{\prime} 20$ long; tarsus 5 long, antaxial fastigial tarsal seta $f t^{\prime \prime} 15$ long, paraxial fastigial tarsal seta $f t^{\prime} 15$ long, paraxial unguinal tarsal seta $u^{\prime} 4$ long, solenidion slightly curved, tapered apically, 6 long; empodium divided, 5 long. 6-rayed. Leg II 18 long; femur 8 long, ventral basifemoral seta $b v$ absent; genu 3 long, antaxial genual seta $l^{\prime \prime}$ absent; tibia 2 long; tarsus 5 long, antaxial fastigial tarsal seta $f t^{\prime \prime} 15$ long, paraxial fastigial tarsal seta $f t^{\prime} 8$ long, paraxial unguinal tarsal seta $u^{\prime} 4$ long, solenidion slightly curved, tapered apically, 6 long; empodium divided, 5 long, 6-rayed. Coxisternal plates: coxisterna I and II with smooth surfaces. Coxisternum I fused, not forming internal coxisternal apodeme. Anterior seta on coxisternum I $1 b 6$ long, 14 apart; proximal seta on coxisternum I $1 a 13$ long, 7 apart; proximal seta on coxisternum II $2 a 16$ long, 18 apart; distance between setae $1 b$ and la 8 long; coxigenital semiannuli 4, smooth. Opisthosoma: with 23 smooth dorsal annuli, 52 microtuberculate ventral annuli. Opisthosomal seta $c 220$ long, 40 apart, on annulus 6; opisthosomal seta $d$ and $e$ absent; opisthosomal seta $f 23$ long, 15 apart, on annulus 46, or 6 from the rear. Opisthosomal seta $h 227$ long; opisthosomal seta $h l$ absent. Genitalia: 20 wide, proximal seta on coxisternum III $3 a 17$ long, 16 apart.

Etymology: the species name by Hong (1996) is for the country where the mite was first found, Thailand.

Type material: female and male paratypes on three slides labeled \#86 and 28 slides labeled \#302.

Host plant and locality: collected from leaves of Bambusa sp. (Phai), Poaceae, Bambuseae, Nonthaburi Province, Thailand, 3 February 1992, collected by A. Chandrapatya; and from Bambusa vulgaris Schrad. ex J.C. Wendl. (Phai lueang), Poaceae, Bambuseae, Amphoe Lum Luk Ka, Pathum Thani Province, Thailand, 27 January 1997; collected by A. Chandrapatya.

Relation to host: vagrant on the lower leaf surface. No apparent damage was observed.

Neocatarhinus namtai replacement name for Neocatarhinus bambusae (Boczek, 1998), junior homonym of Neocatarhinus bambusae Kuang \& Hong, 1990: 367-371.

Rhyncaphytoptus bambusae Boczek, 1998; Boczek \& Chandrapatya, 1998: 140-142, f.5.

Neocatarhinus bambusae (Boczek, 1998), new combination; junior homonym of Neocatarhinus bambusae Kuang \& Hong, 1990.

\section{Redescription:}

FEMALE (Fig.6) ( $\mathrm{n}=9$ ). Body fusiform, 151 (151-199) long, 58 (58-83) wide, (70-75) thick; color in life red or brown. Gnathosoma: projecting downwards, 37 (37-45) long; pedipalp coxal seta ep 2 (2-3) long, dorsal pedipalp genual seta $d$ absent, subapical pedipalp tarsal seta $v$ 2 long, hook-like. Chelicerae 35 (35-48) long, abruptly bent down. Prodorsal shield: 48 (4852) long, 55 (55-70) wide, subtriangular. Scapular setae sc 1 long, on scapular tubercles 18 (18-27) apart, situated 8 (7-11) ahead of rear shield margin, directed centrally. Shield design 


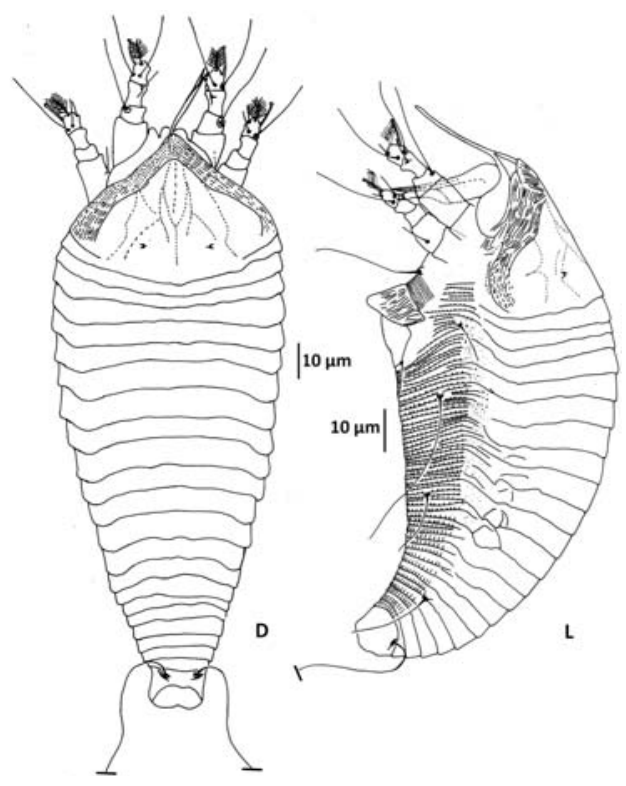

Fig. 6. Neocatarhinus namtai (Boczek)

consists of several indistinct curved lines forming median oval cell and 3-4 longitudinal curved lines on each side, antero-laterally ornamented with numerous short striae. Prodorsal shield frontal lobe rounded anteriorly, extending over gnathosoma, 8 (8-11) long, with 23 (17-23) long filament at the tip. Legs: lacking setae on femur I and genu II. Leg I 30 (30-35) long; femur 10 (10-12) long, ventral basifemoral seta $b v$ absent; genu 6 (4-6) long, antaxial genual seta $l$ " 30 (25-37) long; tibia 7 (7-9) long, paraxial tibial seta $l^{\prime} 13$ (10-15) long, situated antero-laterally on the adaxial surface; tarsus 7 (7-8) long, antaxial fastigial tarsal seta $f t^{\prime \prime} 25$ (22-30) long, paraxial fastigial tarsal seta $f t^{\prime} 22$ (20-30) long, paraxial unguinal tarsal seta $u^{\prime} 7$ (7-10) long, solenidion slightly curved, slight knobbed, 10 (9-10) long; empodium simple, 8 (8-9) long, 7-rayed. Leg II 30 (30-35) long; femur 10 (9-12) long, ventral basifemoral seta $b v 11$ (7-13) long; genu 6 (4-6) long, antaxial genual seta $l^{\prime \prime}$ absent; tibia 7 (6-7) long; tarsus 7 long, antaxial fastigial tarsal seta $f t^{\prime \prime} 29$ (22-30) long, paraxial fastigial tarsal seta $f t^{\prime} 12$ (9-12) long, paraxial unguinal tarsal seta $u^{\prime} 7$ (5-8) long, solenidion almost straight, slightly knobbed, 8 (7-10) long; empodium simple, 8 (8-9) long, 7-rayed. Coxisternal plates: coxisterna I and II with few short lines. Internal coxisternal apodeme a line 13 (13-18) long. Anterior seta on coxisternum I $1 b 10$ (10-14) long, 9 (8-12) apart; proximal seta on coxisternum I 1 a 14 (14-24) long, 6 (5-9) apart; proximal seta on coxisternum II $2 a 38$ (35-55) long, 21 (17-24) apart; distance between setae $1 b$ and $1 a$ (11-13) long; coxigenital semiannuli 6 (6-8), microtuberculate. Opisthosoma with 21 (2122) smooth dorsal annuli. Ventral opisthosoma with 67 (56-69) annuli, microtubercles slightly elongated, situated on rear margin of annuli. Microtubercles more elongated on the last 8 (89) ventral annuli. Dorsal annuli forming a broad middorsal furrow between first 14-15 annuli. Opisthosomal seta $c 216$ (15-30) long, 47 (47-57) apart, on annulus 8 (7-14); opisthosomal 

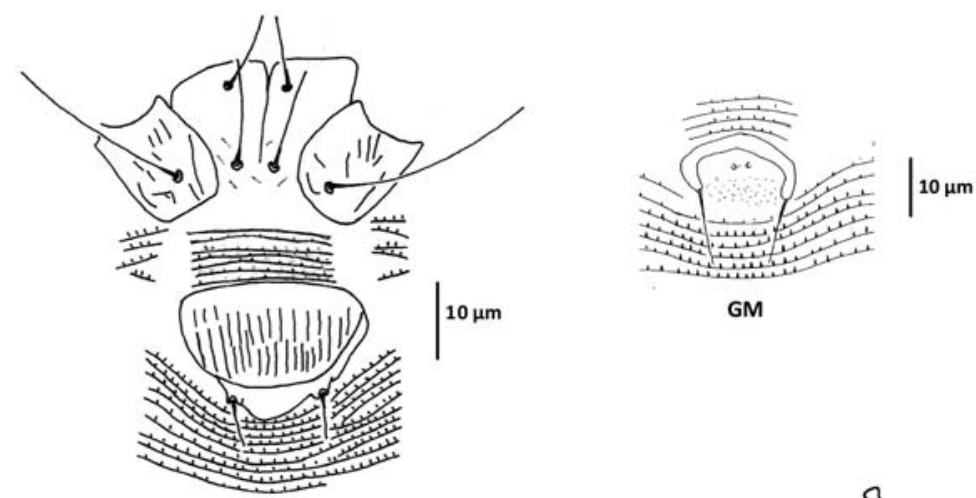

GF
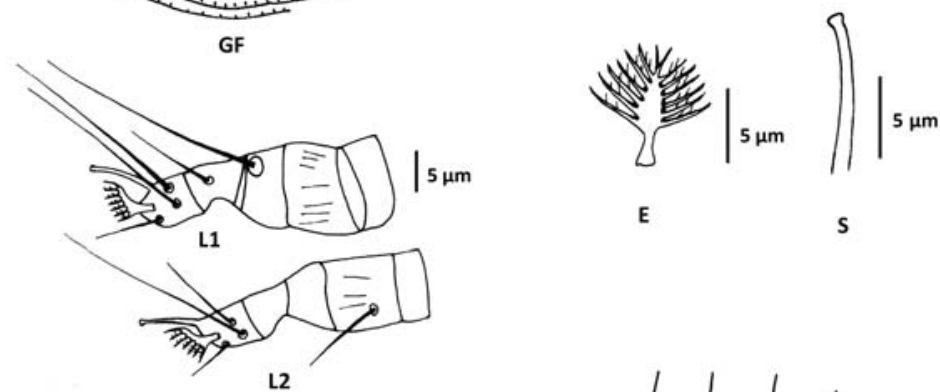

E S

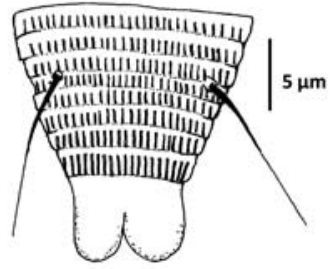

CS

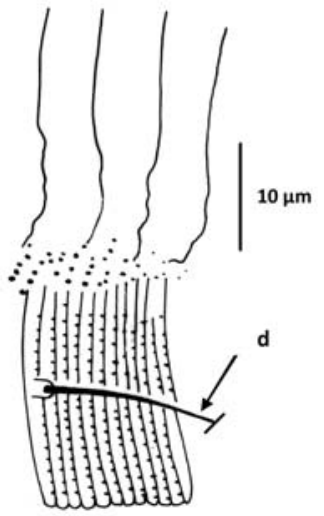

LO

Fig. 6. (Continue) Neocatarhinus namtai (Boczek)

seta $d 46$ (46-85) long, 35 (30-38) apart, on annulus 24 (17-29); opisthosomal seta $e 17$ (1020) long, 15 (14-19) apart, on annulus 45 (38-47); opisthosomal seta $f 22$ (22-35) long, 24 (2330) apart, on annulus 61 (51-64), or 6 (5-6) from rear. Opisthosomal seta $h 275$ (50-80) long; opisthosomal seta $h l$ absent. Genital coverflap: 22 (22-30) wide, 14 (14-21) long with 11-12 broken longitudinal ridges; proximal seta on coxisternum III $3 a$ 8 (6-13) long, 13 (10-13) apart.

MALE $(\mathrm{n}=3)$. 155-160 long, 62-64 wide. Gnathosoma: 38 long; pedipalp coxal seta ep 2 long, dorsal pedipalp genual seta $d$ absent, subapical pedipalp tarsal seta $v 2$ long, hook-like. Chelicerae 35 long. Prodorsal shield: 43-48 long, 54-59 wide, similar to female. Scapular setae 
sc 1-2 long, on scapular tubercles 18-19 apart, situated 10 ahead of rear shield margin, directed centrally. Prodorsal shield frontal lobe 10-11 long. Legs: leg I 26-30 long; femur 10-11 long, ventral basifemoral seta $b v$ absent; genu 4-5 long, antaxial genual seta $l$ " 26-32 long; tibia 7 long, paraxial tibial seta $l^{\prime} 15$ long; tarsus 5-7 long, antaxial fastigial tarsal seta $f t^{\prime \prime}$ 20-23 long, paraxial fastigial tarsal seta $f t^{\prime} 18-22$ long, paraxial unguinal tarsal seta $u^{\prime} 7$ long, solenidion slightly curved, slightly knobbed, 7-10 long; empodium simple, 7-9 long, 7-rayed. Leg II 2528 long; femur 10 long, ventral basifemoral seta $b v$ 10-12 long; genu 4-5 long, antaxial genual seta $l$ " absent; tibia 5-6 long; tarsus 6-7 long, antaxial fastigial tarsal seta $f t$ " 22-25 long, paraxial fastigial tarsal seta $f t^{\prime}$ 7-10 long, paraxial unguinal tarsal seta $u^{\prime} 6$ long, solenidion almost straight, slightly knobbed, 6-8 long; empodium simple 6-7 long, 7-rayed. Coxisternal plates: coxisterna I and II with a few short lines. Internal coxisternal apodeme a line 13-17 long. Anterior seta on coxisternum I $1 b$ 10-12 long, 7-8 apart; proximal seta on coxisternum I 1a 18-20 long, 5-7 apart; proximal seta on coxisternum II $2 a$ 35-39 long, 18-21 apart; distance between seta $1 a$ ad $1 b$ 9-11 long; coxigenital semiannuli 6-7, microtuberculate. Opisthosoma with 21-22 smooth dorsal annuli, 51-54 microtuberculate ventral annuli. Opisthosomal seta $c 2$ 10-15 long, 42-44 apart, on annulus 8-10; opisthosomal seta $d 40-55$ long, 22-24 apart, on annulus 17-20; opisthosomal seta $e$ 12-15 long, 10-14 apart, on annulus 32-35; opisthosomal seta $f$ 17-28 long, 20-23 apart, on annulus 45 (45-48), or 6 from the rear. Opisthosomal seta $h 2$ 35-47 long; opisthosomal seta $h 1$ absent. Genitalia: 15-18 wide, proximal seta on cosisternum III 3 a 12-13 long, 14-15 apart.

Etymology: the species name is from the Thai common name, Phai namtao.

Type material: female and male paratypes on 9 slides labeled \#213 and 18 slides labeled \#785.

Host plant and locality: collected from leaves of Bambusa ventricosa McClure (Phai namtao, Buddha's belly bamboo), Poaceae, Bambuseae, Amphoe Kamphaeng Saen, Nakhon Pathom Province, Thailand, 2 November 1996, collected by A. Chandrapatya; and from Melocanna humilis Roep. ex Trin. (Phai kriap), Poaceae, Bambuseae, Amphoe Hua Hin, Prachuap Khiri Khan Province, Thailand, 12 May 1998, collected by A. Chandrapatya.

Other hosts and distribution: Bambusa sp. (Phai), Poaceae, Bambuseae, Amphoe Mueang, Kamphaeng Phet Province, Thailand, 6 December 1996, collected by A. Chandrapatya (three slides, labeled \# 269.2); Bambusa sp. (Phai), Poaceae, Bambuseae, Amphoe Sikao, Trang Province, Thailand, 29 March 1997, collected by A. Chandrapatya (30 slides, labeled \#415); Bambusa sp. (Phai), Poaceae, Bambuseae, Amphoe Hua Hin, Prachuap Khiri Khan Province, Thailand, 5 June 1998, collected by A. Chandrapatya (two slides labeled \#788); Bambusa vulgaris Schrad. ex J. C. Wendl. (Phai lueang), Poaceae, Bambuseae, Amphoe Si Racha, Chon Buri Province, Thailand, 12 September 1998, collected by A. Chandrapatya (10 slides labeled \#930.2); Dendrocalamus asper (Schult. \& Schult. f.) Backer ex K. Heyne (Phai tong), Poaceae, Bambuseae, Amphoe Bo Rai, Trat Province, Thailand, 19 April 1999, collected by A. Chandrapatya (65 slides, labeled \#1319); Bambusa sp. (Phai), Poaceae, Bambuseae, Amphoe Mae Rim, Chiang Mai Province, Thailand, 8 December 1999, collected by A. Chandrapatya (57 slides, labeled \#1806).

Relation to host: vagrant on both leaf surfaces. No apparent damage was observed.

Diagnosis: this species is close to Neocatarhynus bambusae Kuang and Hong (1990) but can 
be distinguished by shield design, scapular setae $s c$, coxisternal plates and position of setae on coxisternum, and length and position of paraxial tibial setae. Prodorsal shield of N. bambusae with rounded anterior lobe, decorated with incomplete admedian and submedian lines and numerous short, crowded lines between shield sides and admedian lines. Scapular setae $s c$ of $N$. bambusae more distinct than in N. namtai. Both coxisterna ornamented with granules, anterior seta $l b$ on coxisternum I and proximal seta $l a$ on coxisternum I far apart, proximal seta $l a$ on coxisternum I situated ahead of proximal seta $2 a$ on coxisternum II. Paraxial tibial seta $l^{\prime}$ located at $1 / 3$ from upper end, relatively short. In $N$. namtai, prodorsal shield with a long filament at the tip of the rounded prodorsal shield frontal lobe, shield design with indistinct lines forming a medial, oval cell and 3-4 longitudinal curved lines, numerous granules located anterolaterally, scapular seta $s c$ minute. Coxisterna I and II with a few short lines. Anterior seta $l b$ on coxisternum I and proximal setae $1 a$ on coxisternum I situated close to each other, not far apart, the base of anterior seta $1 a$ on coxisternum I and proximal seta $2 a$ on coxisternum II almost lined up in the same row. Paraxial tibial setae $l$ ' located at $1 / 2$ from upper end, setae are long and distinct.

\section{Kolacarus bambusae Boczek, 1998}

Boczek \& Chandrapatya, 1998: 135-136, f.1.

\section{Redescription:}

FEMALE (Fig.7) (n=9). Body fusiform, 148 (135-165) long, 55 (55-70) wide, 61 (60-65) thick; color in life, transparent. Gnathosoma: projecting downwards, 33 (30-38) long; pedipalp coxal seta ep 3 (3-5) long, dorsal pedipalp genual seta $d 7$ (5-7) long. Chelicerae 24 (23-36) long, almost straight. Prodorsal shield: 36 (33-54) long, 53 (53-63) wide, semicircular. Scapular setae $s c$ and tubercles absent. Shield design consists of several short curved lines. Prodorsal shield frontal lobe broad, short, extending over gnathosoma, 1 (1-2) long. Legs: with all setae present. Leg I 28 (28-31) long; femur 8 (8-9) long, ventral basifemoral seta $b v 11$ (11-16) long; genu 5 (5-7) long, antaxial genual seta $l^{\prime \prime} 28$ (25-37) long; tibia 7 (7-8) long, paraxial tibial seta $l^{\prime} 10$ (610) long; tarsus 8 (6-8) long, antaxial fastigial tarsal seta $f t^{\prime \prime} 30$ (23-30) long, paraxial fastigial tarsal seta $f t^{\prime} 30$ (22-30) long, paraxial unguinal tarsal seta $u^{\prime} 3$ (3-5) long, solenidion slightly curved, tapered apically, 8 (7-8) long; empodium simple, 9 (7-10) long, 8-rayed. Leg II 27 (26-31) long; femur 11 (8-11) long, ventral basifemoral seta $b v 9$ (9-20) long; genu 4 (4-5) long, antaxial genual seta $l^{\prime \prime} 8$ (7-10) long; tibia 7 (6-8) long; tarsus 6 (6-7) long, antaxial fastigial tarsal seta $f^{\prime \prime}$ 25 (20-25) long, paraxial fastigial tarsal seta $f t^{\prime} 14$ (8-14) long, paraxial unguinal tarsal seta $u^{\prime} 3$ (3-5) long, solenidion as in leg I, 8 long; empodium simple, 9 (7-10) long, 8-rayed. Coxisternal plates: with numerous short lines on both coxisterna. Internal coxisternal apodeme a line 7 (47) long. Anterior seta on coxisternum I $1 b 9$ (9-12) long, 14 (14-18) apart; proximal seta on coxisternum I la 23 (15-23) long, 7 (7-8) apart; proximal seta on coxisternum II $2 a 27$ (22-35) long, 24 (22-25) apart; distance between setae $1 b$ and $1 a 9$ (6-9) long; coxigenital semiannuli 7 (7-9), microtuberculate. Opisthosoma: with 51 (45-56) dorsal annuli, with faint microtubercles anteriorly and spinelike microtubercles on last (24-32) annuli. Ventral opisthosoma with 74 (6774) annuli, triangular microtubercles situated on rear margin of annuli. Microtubercles elongated on the last 4-5 ventral annuli. Dorsal annuli evenly rounded. Opisthosomal seta $c 240$ (40-60) 


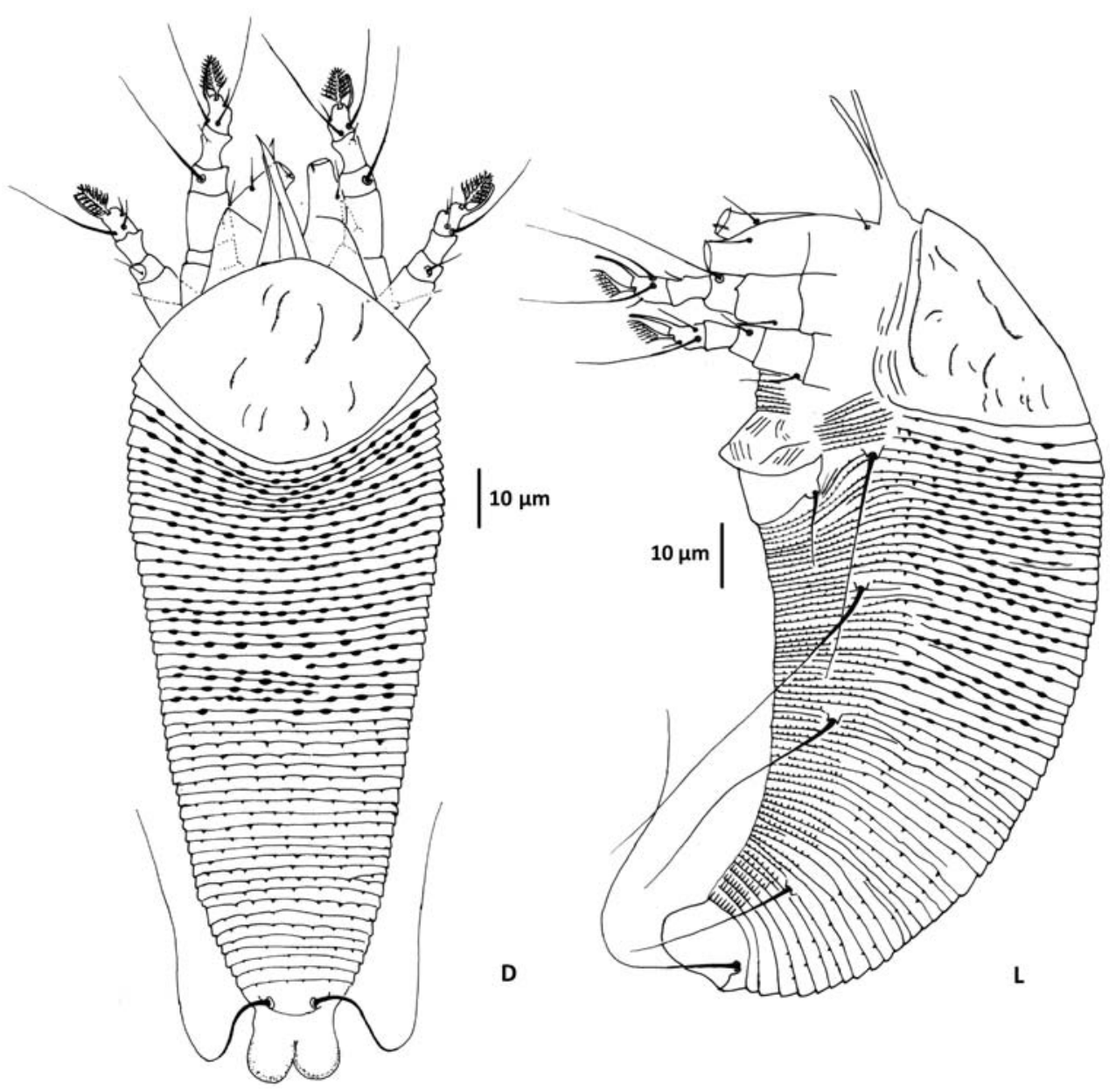

Fig. 7. Kolacarus bambusi (Boczek)

long, 43 (39-44) apart, on annulus 10 (10-13); opisthosomal seta $d 58$ (42-60) long, 32 (28-33) apart, on annulus 23 (23-28); opisthosomal seta $e 44$ (40-55) long, 20 (20-23) apart, on annulus 44 (40-48); opisthosomal seta $f 30$ (28-35) long, 23 (20-25) apart, on annulus 67 (60-67), or 7 (78) from the rear. Opisthosomal seta $h 264$ (50-64) long; opisthosomal seta $h l$ absent. Genital coverflap: 23 (21-24) wide, 18 (15-18) long, with two rows of broken longitudinal ridges; proximal seta on coxisternum III $3 a 26$ (19-26) long, 16 (15-16) apart.

MALE $(\mathrm{n}=1) .140$ long, 58 wide, 64 thick. Gnathosoma: 25 long; pedipalp coxal seta ep 5 long, dorsal pedipalp genual seta $d 4$ long. Chelicerae 28 long. Prodorsal shield: 39 long, 54 wide, similar to female. Scapular setae $s c$ and tubercles absent. Prodorsal shield frontal lobe 2 long. Legs: leg I 30 long; femur 10 long, ventral basifemoral seta $b v 15$ long; genu 5 long, antaxial genual seta $l^{\prime \prime} 28$ long; tibia 8 long, paraxial tibial seta $l^{\prime} 7$ long; tarsus 7 long, antaxial 

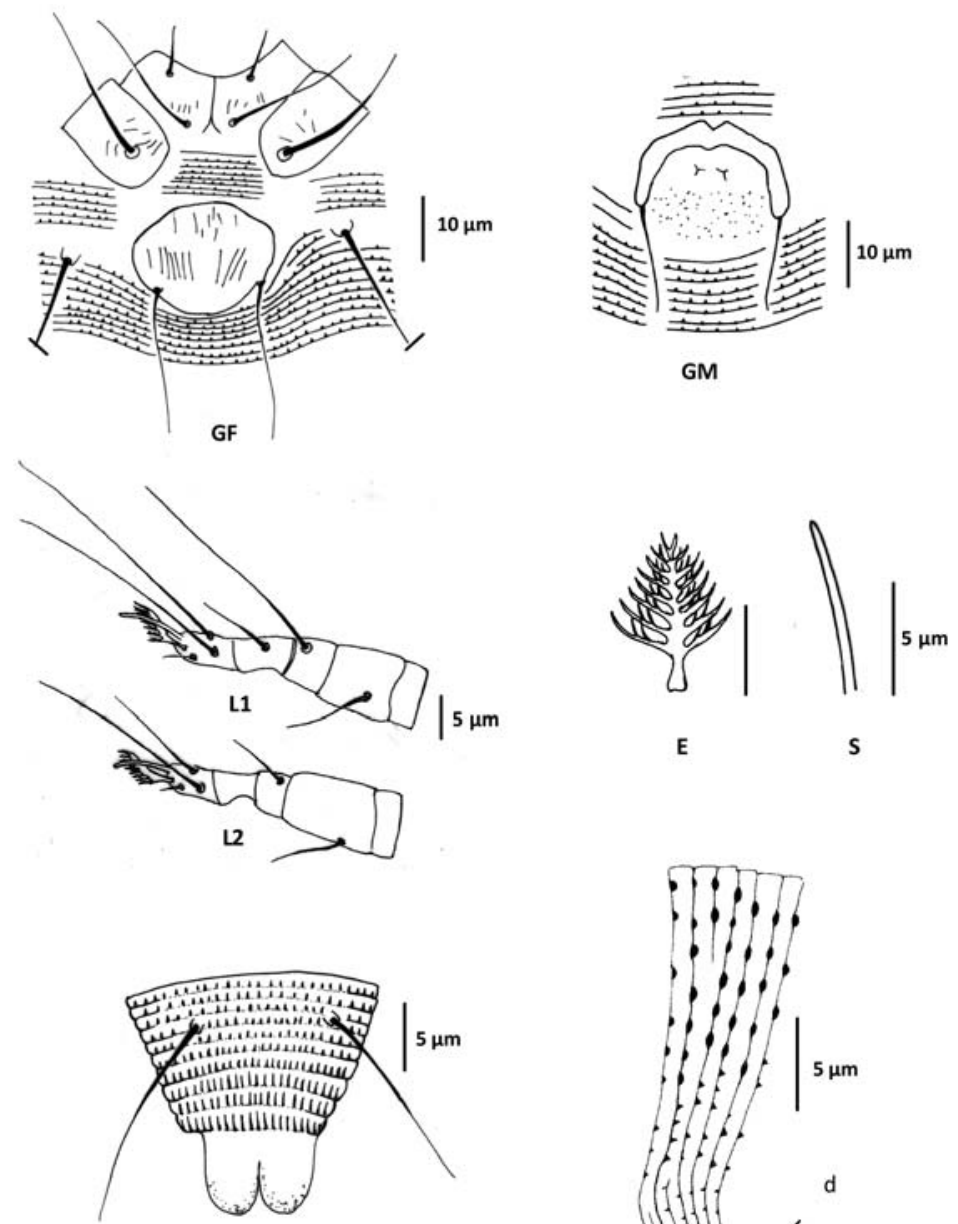

CS

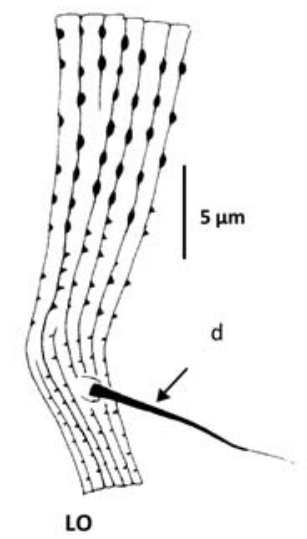

Fig. 7. (Continue) Kolacarus bambusi (Boczek)

fastigial tarsal seta $f t^{\prime \prime} 22$ long, paraxial fastigial tarsal seta $f t^{\prime} 21$ long, paraxial unguinal tarsal seta $u^{\prime} 4$ long, solenidion slightly curved, tapered apically, 7 long; empodium simple, 8 long, 8-rayed. Leg II 26 long; femur 11 long, ventral basifemoral seta bv 16 long; genu 4 long, antaxial genual seta $l^{\prime \prime} 9$ long; tibia 5 long; tarsus 6 long, antaxial fastigial tarsal seta $f t^{\prime \prime} 25$ long, paraxial fastigial tarsal seta $f t^{\prime} 7$ long, paraxial unguinal tarsal seta $u^{\prime} 4$ long, solenidion as in leg I, 7 long; empodium simple, 8 long, 8-rayed. Coxisternal plates: with numerous short lines on both coxisterna. Internal coxisternal apodeme a line 6 long. Anterior seta on coxisternum I $1 b 8$ long, 15 apart; proximal seta on coxisternum I 1 a 15 long, 5 apart; proximal seta on coxisternum 
II $2 a 30$ long, 20 apart; distance between setae $1 b$ and $1 a 6$ long; coxigenital semiannuli 8, microtuberculate. Opisthosoma: with 42 dorsal annuli with faint microtubercles anteriorly and spine-like microtubercles posteriorly, 62 microtuberculate ventral annuli. Opisthosomal seta $c 240$ long, 37 apart, on annulus 12; opisthosomal seta $d 40$ long, 30 apart, on annulus 22; opisthosomal seta $e 30$ long, 20 apart, on annulus 41; opisthosomal seta $f 22$ long, 22 apart, on annulus 54, or 8 from the rear. Opisthosomal seta $h 250$ long; opisthosomal seta $h l$ absent. Genitalia: 20 wide, proximal seta on coxisternum III $3 a 18$ long, 20 apart.

Etymology: named after the plant genus of the original host, Bambusa.

Type material: female paratype on 13 slides labeled \#202.

Host plant and locality: collected from leaves of Bambusa vulgaris Schrad. ex J.C. Wendl. (Phai lueang), Poaceae, Bambuseae, Ban Pae, Amphoe Klaeng, Rayong Province, Thailand, 2 November 1996, collected by A. Chandrapatya.

Other hosts and distribution: Bambusa sp. (Phai), Poaceae, Bambuseae, Amphoe Mueang, Nakhon Pathom Province, Thailand, 30 April 1998, collected by A. Chandrapatya (32 slides labeled \#781); Bambusa multiplex (Lour.) Raeusch. ex Schult. \& Schult. f. (Phai liang), Poaceae, Bambuseae, Amphoe Kamphaeng Saen, Nakhon Pathom Province, Thailand, 14 August 1998, collected by A. Chandrapatya (54 slides labeled \#922); Bambusa vulgaris (Phai lueang), Poaceae, Bambuseae, Amphoe Si Racha, Chon Buri Province, Thailand, 12 September 1998; collected by A. Chandrapatya (11 slides labeled \#930); Bambusa bambos (L.) Voss [excluded], Poaceae, Bambuseae, Amphoe Cha-am, Phetchaburi, Thailand, 23 January 1999, collected by A. Chandrapatya (32 slides labeled \#1133); Bambusa vulgaris, Poaceae, Bambuseae, Amphoe Amphawa, Samut Songkhram Province, Thailand, 20 March 1999, collected by A. Chandrapatya (13 slides labeled \#1257); Bambusa ventricosa McClure (Phai namtao, Buddha's belly bamboo), Poaceae, Bambuseae, Salaya, Amphoe Phutthamonthon, Nakhon Pathom Province, Thailand, 27 April 1999, collected by A. Chandrapatya (34 slides labeled \#1368.1); Bambusa vulgaris (Phai lueang), Poaceae, Bambuseae, Amphoe Mueang, Rayong Province, Thailand, 12 October 1999, collected by A. Chandrapatya (17 slides labeled \#1668); Bambusa blumeana Schult. (Phai si suk), Poaceae, Bambuseae, Amphoe Bang Kruai, Nonthaburi Province, Thailand, 29 March 2000, collected by A. Chandrapatya (50 slides labeled \#1910); Bambusa sp. (Phai), Poaceae, Bambuseae, Amphoe Kamphaeng Saen, Nakhon Pathom Province, Thailand, 30 March 2000, collected by A. Chandrapatya (39 slides labeled \#1936); Dendrocalamus latiflorus Munro (Phai sang nuan), Poaceae, Bambuseae, Amphoe Mueang, Uttaradit Province, Thailand, 10 October 2000, collected by A. Chandrapatya (7 slides labeled \#2078).

Relation to host: vagrant on the lower leaf surface. No apparent damage was observed.

\section{ACKNOWLEDGEMENTS}

A part of this research was partially supported by the Center for Advanced Studies for Agriculture and Food, Institute for Advanced Studies, Kasetsart University under the Higher Education Research Promotion and National Research University Project of Thailand, Office of the Higher Education Commission and Thailand Research Fund (TRF Senior Research Scholar \#RTA4880006). 


\section{REFERENCES}

Amrine J.W.Jr. and Stasny, T.A. (1994) Catalog of the Eriophyoidea (Acarina: Prostigmata) of the world. Indira Publishing House, Michigan, USA, 804 pp.

Amrine, J.W.Jr., T.A. Stasny and C.H.W. Flechtmann (2003) Revised key to world genera of Eriophyoidea (Acari: Prostigmata). Indira Publishing House, USA, 244 pp.

Boczek, J. and A. Chandrapatya.(1998) Studies on eriophyoid mites (Acari: Eriophyoidea). XXII. Acarologia (Paris), 39: 135-142.

Chandrapatya, A. and J. Boczek. (1993) Studies on eriophyid mites (Acari: Eriophyoidea). XII. Bulletin of the Polish Academy of Sciences, Biological Sciences, 41: 45-52.

Chandrapatya, A. and J. Boczek. (2002) Studies on eriophyoid mites (Acari: Eriophyoidea). A-1. Bulletin of the Polish Academy of Sciences, Biological Sciences, 50: 135-147.

ChannaBasavanna, G.P. (1966) A contribution to the knowledge of Indian eriophyid mites (Eriophyoidea: Trombidiformes: Acarina). University of Agricultural Science, Hebbal, Bangalore, India, 154 pp.

de Lillo, E., C. Craemer, J. W. Amrine Jr. and G. Nuzzaci (2010) Recommended procedures and techniques for morphological studies of Eriophyoidea (Acari: Prostigmata). Experimental and Applied Acarology, 51: 283-307.

Hong, X.-Y. (1996) Knorella thailandica, a new name for Knorella bambusae Chandrapatya and a key to world species of Knorella (Acari: Eriophyidae). Systematic and Applied Acarology, 1: 205-206.

Huang, K.-Y. (2001) The eriophyid mites of Taiwan: description of eighty-six species from the Tengchih Area. Bulletin of Natural Museum of Natural Science, 14: 1-84.

Keifer, H.H. (1959) Eriophyid Studies XXVII. Occasional Papers 1. California Department of Agriculture, 18 pp.

Keifer, H.H. (1975) Eriophyid Studies C-10. U.S. Department of Agriculture, Agricultural Research Service, 24 pp.

Keifer, H.H. (1977) Eriophyid Studies C-14. U.S. Department of Agriculture, Agricultural Research Service, 24 pp.

Keifer, H. H. (1978) Eriophyid Studies C-15. U.S. Department of Agriculture, Agricultural Research Service, 24 pp.

Kuang, H.-Y. and Y.-B. Feng (1989) Description of a new genus and three new species of Phyllocoptinae from China (Acariformes: Eriophyidae). Acta Zootaxonomica Sinica, 14: 37-41.

Kuang, H. -Y. and X. Y. Hong (1990) One new genus and three new species of the family Rhyncaphytoptidae (Acari: Eriophyoidea) from the People's Republic of China. Acarologia (Paris), 31(4): 367-371.

Kuang, H. -Y. and W.-X. Zhuo (1987) Two new species and a new record of the genus Abacarus from China (Acariformes: Eriophyidae). Acta Zootaxonomica Sinica, 12: 380-382.

Xue, X.-F. and Z.-Q. Zhang (2009) Eriophyoid mites (Acari: Prostigmata) in Southeast Asia: a synopsis of 104 genera, with an illustrated key to genera and checklist of species. Zootaxa, 2257: 1-128.

\section{Abbreviations:}

$\mathrm{CV}=$ ventral view of cauda, $\mathrm{D}=$ dorsal view of female, $\mathrm{E}=$ empodium, $\mathrm{GF}=$ female genitalia, $\mathrm{GM}=$ male genitalia, $\mathrm{L}=$ lateral view of female, $\mathrm{L} 1=$ leg $\mathrm{I}, \mathrm{L} 2=$ leg $\mathrm{II}, \mathrm{S}=$ solenidion, $\mathrm{LO}=$ lateral opisthosoma 